\title{
STOCHASTIC RADON OPERATORS IN POROUS MEDIA HYDRODYNAMICS
}

\author{
BY \\ GEORGE CHRISTAKOS A.ND DIONISSIOS T. HRISTOPULOS \\ University of North Carolina, Chapel Hill, N.C.
}

\begin{abstract}
A space transformation approach is established to study partial differential equations with space-dependent coefficients modelling porous media hydrodynamics. The approach reduces the original multi-dimensional problem to the one-dimensional space and is developed on the basis of Radon and Hilbert operators and generalized functions. In particular, the approach involves a generalized spectral decomposition that allows the derivation of space transformations of random field products. A Plancherel representation highlights the fact that the space transformation of the product of random fields inherently contains integration over a "dummy" hyperplane. Space transformation is first examined by means of a test problem, where the results are compared with the exact solutions obtained by a standard partial differential equation method. Then, exact solutions for the flow head potential in a heterogeneous porous medium are derived. The stochastic partial differential equation describing three-dimensional porous media hydrodynamics is reduced into a one-dimensional integro-differential equation involving the generalized space transformation of the head potential. Under certain conditions the latter can be further simplified to yield a first-order ordinary differential equation. Space transformation solutions for the head potential are compared with local solutions in the neighborhood of an expansion point which are derived by using finite-order Taylor series expansions of the hydraulic log-conductivity.
\end{abstract}

1. Introduction. First of all, let us introduce some notations. By $\mathbf{R}^{n}$ we denote the $n$-dimensional Euclidean space, $\mathbf{s}=\left(s_{1}, \ldots, s_{n}\right) \in \mathbf{R}^{n}$ is the position vector, $\boldsymbol{\theta}=$ $\left(\theta_{1}, \ldots, \theta_{n}\right)$ is the unit vector, and $S_{n}=\frac{2 \pi^{n / 2}}{\Gamma\left(\frac{n}{2}\right)}$ is the surface area of the $n$-dimensional sphere ( $\Gamma$ is the gamma function). Integrations are carried out over the whole range of a variable, unless other limits are indicated. Given a function $f(\mathbf{s})$ on $\mathbf{R}^{n}$, consider

Received March 29, 1994.

1991 Mathematics Subject Classification. Primary 86A05, 76S05, 60G60.

Key words and phrases. Hydrodynamics, Radon transforms, Hilbert transform, random fields, space transformations, generalized functions.

Research has been supported by grants from the Army Research Office (contract no. DAAL03-92-G0111), and the National Institute of Environmental Health Sciences, Research Triangle Park, North Carolina (contract no. P42 ES05948-02). 
(i) the mapping

$$
\mathcal{R}: f(\mathbf{s}) \rightarrow \int d \mathbf{s} f(\mathbf{s}) \delta(\sigma-\mathbf{s} \cdot \boldsymbol{\theta})
$$

where $\sigma \in R^{1}$ and $\delta$ is the delta function. The $f(\mathbf{s})$ are suitable functions (ordinary or generalized) so that the integral in Eq. (1.1) exists. For example, $f(\mathbf{s})$ may belong to the Schwartz space. Equation (1.1) is known as the Radon transform (e.g., [7]) or as the plane wave integral (e.g., [8]). Also consider

(ii) the operator

$$
\Omega=\frac{(-1)^{m-1}}{2(2 \pi)^{2 m-1}} \begin{cases}-\frac{S_{2 m+1}}{2 \pi} \frac{\partial^{2 m}}{\partial \sigma^{2 m}}[\cdot], & \text { if } n=2 m+1, \\ S_{2 m} \mathcal{H}\left\{\frac{\partial^{2 m-1}}{\partial \sigma^{2 m-1}}[\cdot]\right\}, & \text { if } n=2 m,\end{cases}
$$

where $\mathcal{H}$ denotes the Hilbert transform.

In general, space transformations (ST) are mathematical operations that reduce a multi-dimensional space to a uni-dimensional space. In stochastic analysis ST can be defined in terms of random fields (RF) or by means of their statistical moments. The usefulness of ST in the study of environmental systems has been discussed extensively, e.g., in [2], [3], and [4]. In the following, the RF are considered in the sense of [9].

Definition 1.1. Consider the $n$-dimensional RF $X_{n}(\mathbf{s})$. The ST operators $T_{n}^{1}$ and $\Psi_{n}^{1}$, that reduce the $n$-dimensional RF to a uni-dimensional RF by means of the mapping (1.1) and the operator (1.2), are defined as follows:

$$
T_{n}^{1}\left[X_{n}\right](\sigma, \boldsymbol{\theta})=\widehat{X}_{1 . \boldsymbol{\theta}}(\sigma)
$$

where $T_{n}^{1}[\cdot]=\mathcal{R}[\cdot]$; and

$$
\Psi_{n}^{1}\left[X_{n}\right](\sigma, \boldsymbol{\theta})=\Omega T_{n}^{1}\left[X_{n}\right](\sigma, \boldsymbol{\theta})=X_{1 . \boldsymbol{\theta}}(\sigma)
$$

The ST, $T_{n}^{1}$, and $\Psi_{n}^{1}$ are considered as completely defined if the integral expressions in Eqs. (1.3) and (1.4) are known for all $\boldsymbol{\theta}$.

Some properties of the ST are worth exploiting. The $T_{n}^{1}$ consists of infinitely many integral transforms with the delta functions as their kernels. The $T_{n}^{1}$ of a function may also be considered as a homogeneous function of degree -1 on projective space. The ST operators (1.3) and (1.4) can be inverted as follows:

$$
\Psi_{1}^{n}\left[X_{1, \boldsymbol{\theta}}\right](\mathbf{s})=S_{n}^{-1} \int_{S_{n}} d \boldsymbol{\theta} X_{1, \boldsymbol{\theta}}(\mathbf{s} \cdot \boldsymbol{\theta})=X_{n}(\mathbf{s})
$$

and

$$
T_{1}^{n}\left[\widehat{X}_{1, \boldsymbol{\theta}}\right](\mathbf{s})=\Psi_{1}^{n} \Omega\left[\widehat{X}_{1, \boldsymbol{\theta}}\right](\mathbf{s})=X_{n}(\mathbf{s})
$$

In the spectral domain $\Phi \subseteq \mathbf{R}^{n}$, the ST turn out to have simple algebraic forms. Indeed, for the $T_{n}^{1}$ we have

$$
T_{n}^{1}\left[\widetilde{X}_{n}\right](\omega, \boldsymbol{\theta})=\widetilde{X}_{n}(\mathbf{w})=\tilde{\hat{X}}_{1, \boldsymbol{\theta}}(\omega)
$$


along the wavevector $\mathbf{w}=\omega \boldsymbol{\theta} \in \Phi$, where $\omega=|\mathbf{w}|$ and $\tilde{X}$ denotes the Fourier transform of $X$; and, for the $T_{1}^{n}$,

$$
T_{1}^{n}\left[\tilde{\hat{X}}_{1, \boldsymbol{\theta}}\right](\mathbf{w})=\tilde{\hat{X}}_{1, \boldsymbol{\theta}}(\omega)=\tilde{X}_{n}(\mathbf{w})
$$

is valid. Similarly, for the $\Psi_{n}^{1}$ and $\Psi_{1}^{n}$ it can be shown that

$$
\Psi_{n}^{1}\left[\widetilde{X}_{n}\right](\omega, \boldsymbol{\theta})=\frac{S_{n}|\omega|^{n-1}}{(2 \pi)^{n-1}} \widetilde{X}_{n}(\mathbf{w})=\widetilde{X}_{1, \boldsymbol{\theta}}(\omega),
$$

and

$$
\Psi_{1}^{n}\left[\widetilde{X}_{1, \boldsymbol{\theta}}\right](\mathbf{w})=\frac{(2 \pi)^{n-1}}{S_{n}|\omega|^{n-1}} \widetilde{X}_{1, \boldsymbol{\theta}}(\omega)=\widetilde{X}_{n}(\mathbf{w})
$$

respectively.

In the context of stochastic partial differential equations (PDE) with space-dependent coefficients, of particular importance is the development of ST expressions for RF products. This type of PDE governs subsurface porous media hydrodynamics and is thus very important in environmental applications. In Sec. 2 we derive expressions for ST of the product of two RF by means of generalized function representations. In Sec. 3 a Plancherel equation is established for the ST operations. Section 4 considers certain series expansions of ST. In Sec. 5 a test example is examined where solutions are obtained by means of the ST approach and are compared with the exact solutions derived by a standard PDE method. In Sec. 6 solutions of three-dimensional groundwater flow in porous formations are derived in terms of solutions of a one-dimensional model along various directions in space. The realistic scenario of point-like boundary conditions is considered. Also, the case of Neumann boundary conditions on a spherical domain is examined. In Sec. 7 the ST solutions are compared with local solutions obtained by means of multidimensional series expansions. Section 8 deals with numerical considerations and stochastic moments solutions concerning the application of the ST method in realistic situations of porous media hydrodynamics.

2. Space transformations of random field products and gradients. In the following we will focus in the $\mathbf{R}^{3}$ space. However, the extension of the analysis in the $\mathbf{R}^{n}$ space is straightforward.

Let the $\mathrm{RF}_{3}(\mathbf{S})$ be expressed as

$$
Y_{3}(\mathbf{s})=\int d \mathbf{z} Y_{3}(\mathbf{z}) \delta(\mathbf{s}-\mathbf{z})
$$

By defining the generalized RF (in the sense, e.g., of [6])

$$
Y_{3 \mathbf{z}}(\mathbf{s})=\delta(\mathbf{s}-\mathbf{z}) Y_{3}(\mathbf{z})
$$

Eq. (2.1) can be written as

$$
Y_{3}(\mathbf{s})=\int d \mathbf{z} Y_{3 \mathbf{z}}(\mathbf{s})
$$


LEMma 2.1. The generalized $\mathrm{RF} Y_{3 \mathbf{z}}(\mathbf{s})$ has a spectral amplitude $\tilde{Y}_{3 \mathbf{z}}(\mathbf{w})$ that admits the following generalized spectral decomposition:

$$
\tilde{Y}_{3 \mathbf{z}}\left(\mathbf{w}-\mathbf{w}^{\prime}\right)=e^{-i \mathbf{w}^{\prime} \cdot \mathbf{z}} \tilde{Y}_{3 \mathbf{z}}(\mathbf{w})
$$

Proof. The Fourier transform of $Y_{3 \mathbf{z}}(\mathbf{s})$ in Eq. (2.2) with respect to $\mathbf{s}$ is $\widetilde{Y}_{3 \mathbf{z}}(\mathbf{w})=$ $Y_{3}(\mathbf{z}) e^{i \mathbf{w} \cdot \mathbf{z}}$, which clearly satisfies the spectral decomposition property $(2.4)$.

The generalized field

$$
\widehat{Y}_{1, \boldsymbol{\theta}, \mathbf{z}}(\sigma)=T_{3}^{1}\left[Y_{3 \mathbf{z}}\right](\sigma, \boldsymbol{\theta})
$$

may be considered as the ST of the generalized $\mathrm{RF} Y_{3 \mathbf{z}}(\mathbf{s})$ or the generalized $\mathrm{ST}$ of the ordinary $\mathrm{RF} Y_{3}(\mathbf{s})$. Clearly,

$$
\widehat{Y}_{1, \boldsymbol{\theta}}(\sigma)=\int d \mathbf{z} \widehat{Y}_{1, \boldsymbol{\theta}, \mathbf{z}}(\sigma)
$$

Moreover, the Fourier transform of Eq. (2.1) can be written as $\tilde{Y}_{3}(\mathbf{w})=\int d \mathbf{z} \widetilde{Y}_{3 \mathbf{z}}(\mathbf{w})$, which leads to the following integral representation:

$$
\widetilde{Y}_{3}\left(\mathbf{w}-\mathbf{w}^{\prime}\right)=\int d \mathbf{z} e^{-i \mathbf{w}^{\prime} \cdot \mathbf{z}} \widetilde{Y}_{3 \mathbf{z}}(\mathbf{w}) .
$$

The analysis above leads us to the following fundamental

Proposition 2.1. Consider two RFs, $X_{3}(\mathbf{s})$ and $Y_{3}(\mathbf{s})$. The $T_{3}^{1}$ transformation of their product is given by

$$
T_{3}^{1}\left[X_{3} Y_{3}\right](\sigma, \boldsymbol{\theta})=\int d \mathbf{z} X_{3}(\mathbf{z}) \widehat{Y}_{1, \boldsymbol{\theta} . \mathbf{z}}(\sigma)
$$

Proof. The $T_{3}^{1}$ of a product of two RFs, $X_{3}(\mathbf{s})$ and $Y_{3}(\mathbf{s})$, can be written as

$$
T_{3}^{1}\left[X_{3} Y_{3}\right](\sigma, \boldsymbol{\theta})=\frac{1}{(2 \pi)^{4}} \int d \omega e^{-i \omega s \cdot \boldsymbol{\theta}} \int d \mathbf{w}^{\prime} \widetilde{X}_{3}\left(\mathbf{w}^{\prime}\right) \tilde{Y}_{3}\left(\mathbf{w}-\mathbf{w}^{\prime}\right) .
$$

It should be noted that $\mathbf{w}=\omega \boldsymbol{\theta}$ is a wavevector constrained to point in the direction $\boldsymbol{\theta}$, whereas $\mathbf{w}^{\prime} \in \Phi$. In light of the analysis above, the $T_{3}^{1}$ is given by

$$
\begin{aligned}
T_{3}^{1}\left[X_{3} Y_{3}\right](\sigma, \boldsymbol{\theta}) & =\frac{1}{(2 \pi)^{4}} \int d \omega e^{-i \omega s \cdot \boldsymbol{\theta}} \int d \mathbf{w}^{\prime} \widetilde{X}_{3}\left(\mathbf{w}^{\prime}\right) \widetilde{Y}_{3}\left(\omega \boldsymbol{\theta}-\mathbf{w}^{\prime}\right) \\
& =\frac{1}{(2 \pi)^{4}} \int d \omega e^{-i \omega \sigma} \int d \mathbf{w}^{\prime} \widetilde{X}_{3}\left(\mathbf{w}^{\prime}\right) \int d \mathbf{z} \widetilde{Y}_{3 \mathbf{z}}\left(\omega \boldsymbol{\theta}-\mathbf{w}^{\prime}\right) \\
& =\frac{1}{(2 \pi)^{4}} \int d \omega e^{-i \omega \sigma} \int d \mathbf{w}^{\prime} \widetilde{X}_{3}\left(\mathbf{w}^{\prime}\right) \int d \mathbf{z} e^{-i \mathbf{w}^{\prime} \cdot \mathbf{z}} \widetilde{Y}_{3 \mathbf{z}}(\omega \boldsymbol{\theta}) \\
& =\int d \mathbf{z} \int \frac{d \omega}{2 \pi} e^{-i \omega \sigma} \tilde{\hat{Y}}_{1, \boldsymbol{\theta}, \mathbf{z}}(\omega) \int \frac{d \mathbf{w}^{\prime}}{(2 \pi)^{3}} e^{-i \mathbf{w}^{\prime} \cdot \mathbf{z}} \widetilde{X}_{3}\left(\mathbf{w}^{\prime}\right) \\
& =\int d \mathbf{z} X_{3}(\mathbf{z}) \widehat{Y}_{1, \boldsymbol{\theta}, \mathbf{z}}(\sigma) .
\end{aligned}
$$

This completes the proof.

Regarding the inverse operator $T_{1}^{3}$ of the product $\widetilde{X}_{1, \boldsymbol{\theta}}(\sigma) \widehat{Y}_{1, \boldsymbol{\theta}}(\sigma)$, a useful expression in the spectral domain is suggested by 
Proposition 2.2. The inverse operator $T_{1}^{3}$ of the product $\widehat{X}_{1, \boldsymbol{\theta}}(\sigma) \widehat{Y}_{1, \boldsymbol{\theta}}(\sigma)$ in the spectral domain is as follows:

$$
T_{1}^{3}\left[\widehat{X}_{1, \boldsymbol{\theta}} \widehat{Y}_{1, \boldsymbol{\theta}}\right](\mathbf{w})=\frac{1}{4 \pi} \int d p \widetilde{X}_{3}\left(\frac{\mathbf{w}}{|\mathbf{w}|} p\right) \tilde{Y}_{3}\left(\mathbf{w}-\frac{\mathbf{w}}{|\mathbf{w}|} p\right),
$$

which relates the spectral $T_{1}^{3}$ of the product of two spatial $T_{3}^{1}$ with the one-dimensional integration of the product of the corresponding spectral $T_{1}^{3}$.

Proof. It is valid that

$$
T_{1}^{3}\left[\widehat{X}_{1, \boldsymbol{\theta}} \widehat{Y}_{1, \boldsymbol{\theta}}\right](\mathbf{s})=\iint d \mathbf{s}^{\prime} d \mathbf{s}^{\prime \prime} X_{3}\left(\mathbf{s}^{\prime}\right) Y_{3}\left(\mathbf{s}^{\prime \prime}\right) \Phi\left(\mathbf{s}, \mathbf{s}^{\prime}, \mathbf{s}^{\prime \prime}\right)
$$

where

$$
\Phi\left(\mathbf{s}, \mathbf{s}^{\prime}, \mathbf{s}^{\prime \prime}\right)=-\frac{\nabla_{s}^{2}}{2(2 \pi)^{3}} \int d \boldsymbol{\theta} \int d \omega \int d p e^{i \omega \boldsymbol{\theta} \cdot\left(\mathbf{s}^{\prime \prime}-\mathbf{s}\right)} e^{i p \boldsymbol{\theta} \cdot\left(\mathbf{s}^{\prime}-\mathbf{s}^{\prime \prime}\right)} .
$$

Working in the spectral domain,

$$
\begin{aligned}
F T\left\{T_{1}^{3}\left[\widehat{X}_{1, \boldsymbol{\theta}} \widehat{Y}_{1, \boldsymbol{\theta}}\right]\right\} & =\int d \mathbf{s} e^{i \omega \boldsymbol{\theta} \cdot s} \iint d \mathbf{s}^{\prime} d \mathbf{s}^{\prime \prime} X_{3}\left(\mathbf{s}^{\prime}\right) Y_{3}\left(\mathbf{s}^{\prime \prime}\right)\left(-\frac{1}{2(2 \pi)^{3}}\right) \\
& \int d \boldsymbol{\theta} \int d q \int d p e^{i p \boldsymbol{\theta} \cdot\left(\mathbf{s}^{\prime}-\mathbf{s}^{\prime \prime}\right)} \nabla_{\mathbf{s}}^{2} e^{i \omega \boldsymbol{\theta} \cdot\left(\mathbf{s}^{\prime \prime}-\mathbf{s}\right)} \\
& =\left(-\frac{1}{2(2 \pi)^{3}}\right) \iint d \mathbf{s}^{\prime} d \mathbf{s}^{\prime \prime} X_{3}\left(\mathbf{s}^{\prime}\right) Y_{3}\left(\mathbf{s}^{\prime \prime}\right) e^{i p \boldsymbol{\theta} \cdot \mathbf{s}^{\prime}} e^{i(q-p) \boldsymbol{\theta} \cdot \mathbf{s}^{\prime \prime}} \\
& \int d \boldsymbol{\theta} \int\left(-q^{2}\right) d q \int d p \int d \mathbf{s} e^{i \mathbf{w} \cdot \mathbf{s}} e^{i q \boldsymbol{\theta} \cdot \mathbf{s}} \\
& =\frac{1}{2(2 \pi)^{3}} \int d \boldsymbol{\theta} \int q^{2} d q \int d p \int d \mathbf{s} e^{i \mathbf{s} \cdot(\mathbf{w}-q \boldsymbol{\theta})} \widetilde{X}_{3}(p \boldsymbol{\theta}) \widetilde{Y}_{3}[(p-q) \boldsymbol{\theta}]
\end{aligned}
$$

where FT denotes the Fourier transform, $\int d \mathbf{s} e^{i \mathbf{s} \cdot(\mathbf{w}-q \boldsymbol{\theta})}=(2 \pi)^{3} \delta(\mathbf{w}-q \boldsymbol{\theta})$, and $\int_{0}^{\infty} d q q^{2} \gamma(\mathbf{w}, q)=1$, with

$$
\gamma(\mathbf{w}, q)=\int d \boldsymbol{\theta} \delta(\mathbf{w}-q \boldsymbol{\theta})=\frac{1}{q^{2}} \delta(|\mathbf{w}|-q)
$$

In view of Eq. (2.13), Eq. (2.12) becomes

$$
\begin{aligned}
F T\left\{T_{1}^{3}\left[\widehat{X}_{1, \boldsymbol{\theta}} \widehat{Y}_{1, \boldsymbol{\theta}}\right]\right\}(\mathbf{w}) & =\frac{1}{4 \pi} \int d p \int_{0}^{\infty} d q \delta(|\mathbf{w}|-q) \tilde{X}_{3}\left(\frac{p}{q} \mathbf{w}\right) \tilde{Y}_{3}\left(\mathbf{w}-\frac{p}{q} \mathbf{w}\right) \\
& =\frac{1}{4 \pi} \int d p \widetilde{X}_{3}\left(\frac{p}{q} \mathbf{w}\right) \widetilde{Y}_{3}\left(\mathbf{w}-\frac{p}{|\mathbf{w}|} \mathbf{w}\right)
\end{aligned}
$$

Finally, using the fact that $F T\left[\widehat{X}_{1, \boldsymbol{\theta}} \widehat{Y}_{1, \boldsymbol{\theta}}\right](\omega, \boldsymbol{\theta})=F T\left\{T_{1}^{3}\left[\widehat{X}_{1, \boldsymbol{\theta}} \widehat{Y}_{1, \boldsymbol{\theta}}\right]\right\}(\mathbf{w})$ at $\mathbf{w}=\omega \boldsymbol{\theta}$, Eq. (2.14) leads to Eq. (2.10). 
Some formulas expressing ST of RF gradients are derived below for future use. In the case of ordinary RF the derivations are straightforward. For example, the $T_{3}^{1}$ of the $i$ th component gradient of an $\mathrm{RF} X_{3}(\mathbf{s})$ can be expressed in terms of the slope of its $T_{3}^{1}$ as

$$
T_{3}^{1}\left[\frac{\partial X_{3}}{\partial s_{i}}\right](\sigma, \boldsymbol{\theta})=\theta_{i} \frac{\partial \widehat{X}_{1, \boldsymbol{\theta}}(\sigma)}{\partial \sigma}
$$

and

$$
\Psi_{3}^{1}\left\{\frac{\partial X_{3}}{\partial s_{i}}\right\}(\sigma, \boldsymbol{\theta})=\Omega\left\{T_{3}^{1}\left[\frac{\partial X_{3}(\mathbf{s})}{\partial s_{i}}\right](\sigma, \boldsymbol{\theta})\right\}=\theta_{i} \frac{\partial X_{1, \boldsymbol{\theta}}(\sigma)}{\partial \sigma} .
$$

The expressions for the gradients of generalized RF are somehow more involved. For example, the $T_{3}^{1}$ of the gradient of $X_{3}(\mathbf{s}) \delta(\sigma-\mathbf{s} \cdot \boldsymbol{\theta})$ is (e.g., [4])

$$
T_{3}^{1}\left[\frac{\partial X_{3}(\mathbf{s}) \delta(\sigma-\mathbf{s} \cdot \boldsymbol{\theta})}{\partial s_{i}}\right]\left(p, \boldsymbol{\theta}^{\prime}\right)=\theta_{i}^{\prime} \frac{\partial \widehat{X}_{1}\left(\sigma, \boldsymbol{\theta}, p, \boldsymbol{\theta}^{\prime}\right)}{\partial p},
$$

where $\widehat{X}_{1}\left(\sigma, \boldsymbol{\theta}, p, \boldsymbol{\theta}^{\prime}\right)=T_{3}^{1}\left[X_{3}(\mathbf{s}) \delta(\sigma-\mathbf{s} \cdot \boldsymbol{\theta})\right]\left(p, \boldsymbol{\theta}^{\prime}\right)$ is a function of a double projection on two separate direction vectors.

3. The Plancherel equation for space transformations. Before proceeding with the Plancherel analysis of ST operators, the following lemma is needed.

LEMma 3.1. Let $Y_{3}^{\prime}(-\mathbf{s})=Y_{3}(\mathbf{s})$. The $T_{3}^{1}$ and $\Psi_{3}^{1}$ are such that $\widehat{Y}_{1, \boldsymbol{\theta}}^{\prime}(-\sigma)=\widehat{Y}_{1, \boldsymbol{\theta}}(\sigma)$ and $Y_{1, \boldsymbol{\theta}}^{\prime}(-\sigma)=Y_{1, \boldsymbol{\theta}}(\sigma)$, respectively.

Proof. The $T_{3}^{1}$ gives

$$
\begin{aligned}
\widehat{Y}_{1, \boldsymbol{\theta}}^{\prime}(-\sigma) & =\int_{-\infty}^{\infty} Y_{3}^{\prime}(\mathbf{s}) \delta(-\sigma-\mathbf{s} \cdot \boldsymbol{\theta}) d \mathbf{s}=\int_{-\infty}^{\infty} Y_{3}(-\mathbf{s}) \delta(-\sigma-\mathbf{s} \cdot \boldsymbol{\theta}) d \mathbf{s} \\
& =-\int_{\infty}^{-\infty} Y_{3}(\mathbf{s}) \delta(-\sigma+\mathbf{s} \cdot \boldsymbol{\theta}) d \mathbf{s}=\int_{-\infty}^{\infty} Y_{3}(\mathbf{s}) \delta(\sigma-\mathbf{s} \cdot \boldsymbol{\theta}) d \mathbf{s},
\end{aligned}
$$

for $\delta(\alpha)=\delta(-\alpha)$. Hence, the $T_{3}^{1}$ satisfies $\widehat{Y}_{1, \boldsymbol{\theta}}^{\prime}(-\sigma)=\widehat{Y}_{1, \boldsymbol{\theta}}(\sigma)$, and the $\Psi_{3}^{1}$ satisfies $Y_{1, \theta}^{\prime}(-\sigma)=Y_{1, \theta}(\sigma)$.

Proposition 3.1. The Plancherel equation involves the product of two different types of ST as follows:

$$
\int X_{3}(\mathbf{s}) Y_{3}(\mathbf{s}) d \mathbf{s}=\Psi_{1}^{3}\left[\int \widehat{X}_{1 . \boldsymbol{\theta}}(\sigma) Y_{1 . \boldsymbol{\theta}}(\sigma) d \sigma\right],
$$

where $\widehat{X}_{1, \boldsymbol{\theta}}(\sigma)=T_{3}^{1}\left[X_{3}\right](\sigma, \boldsymbol{\theta})$.

Proof. The convolution of the RFs, $X_{3}(\mathbf{s})$ and $Y_{3}^{\prime}(\mathbf{s})$, which are both assumed to admit ST representations, is given by $F_{3}\left(\mathbf{s}^{\prime}\right)=\int X_{3}(\mathbf{s}) Y_{3}^{\prime}\left(\mathbf{s}^{\prime}-\mathbf{s}\right) d \mathbf{s}$. The Fourier transform of this convolution is $\widetilde{F}_{3}\left(\mathbf{w}^{\prime}\right)=\widetilde{X}_{3}\left(\mathbf{w}^{\prime}\right) \widetilde{Y}_{3}^{\prime}\left(\mathbf{w}^{\prime}\right)$, which in light of Eqs. (1.7) and (1.8) above can be written as

$$
\tilde{\hat{F}}_{1 . \boldsymbol{\theta}}\left(\mathbf{w}^{\prime}\right)=\tilde{\hat{X}}_{1, \boldsymbol{\theta}}\left(\mathbf{w}^{\prime}\right) \tilde{\hat{Y}}_{1, \boldsymbol{\theta}}^{\prime}\left(\mathbf{w}^{\prime}\right) .
$$

Using $T_{1}^{3}$, the $F_{3}\left(\mathbf{s}^{\prime}\right)$ can also be expressed as

$$
\left.F_{3}\left(\mathbf{s}^{\prime}\right)\right|_{\mathbf{s}^{\prime}=0}=\Psi_{1}^{n}\left\{\Omega\left[\widehat{F}_{1, \boldsymbol{\theta}}\left(\sigma^{\prime}\right)\right]_{\sigma^{\prime}=0}\right\} .
$$


The inverse Fourier transform of Eq. (3.2) is

$$
\widehat{F}_{1, \boldsymbol{\theta}}\left(\sigma^{\prime}\right)=\int \widehat{X}_{1, \boldsymbol{\theta}}(\sigma) \widehat{Y}_{1, \boldsymbol{\theta}}^{\prime}\left(\sigma^{\prime}-\sigma\right) d \sigma
$$

which is substituted into Eq. (3.3) so that

$$
F_{3}(0)=\Psi_{1}^{n}\left\{\int \widehat{X}_{1, \boldsymbol{\theta}}(\sigma) \Omega\left[\widehat{Y}_{1, \boldsymbol{\theta}}\left(\sigma^{\prime}-\sigma\right)\right]_{\sigma^{\prime}=0} d \sigma\right\}
$$

Since $Y_{1, \boldsymbol{\theta}}^{\prime}\left(\sigma^{\prime}\right)=\Omega\left[\widehat{Y}_{1, \boldsymbol{\theta}}^{\prime}\left(\sigma^{\prime}\right)\right]$

$$
\int X_{3}(\mathbf{s}) Y_{3}^{\prime}(-\mathbf{s}) d \mathbf{s}=\Psi_{1}^{n}\left[\int \widehat{X}_{1, \boldsymbol{\theta}}(\sigma) Y_{1, \boldsymbol{\theta}}^{\prime}(-\sigma) d \sigma\right]
$$

Finally, in light of Lemma 3.1, Eq. (3.6) leads to Eq. (3.1).

COROLlary 3.1. The following expression is valid for the $T_{3}^{1}$ of the product of two RFs, $X_{3}(\mathbf{s})$ and $Y_{3}(\mathbf{s})$,

$$
T_{3}^{1}\left[X_{3} Z_{3}\right](\sigma, \boldsymbol{\theta})=\Psi_{1}^{3}\left[\int X_{1, \boldsymbol{\theta}^{\prime}}(p) \widehat{Z}_{1}\left(\sigma, \boldsymbol{\theta}, p, \boldsymbol{\theta}^{\prime}\right) d p\right](\sigma, \boldsymbol{\theta})
$$

where $\widehat{Z}_{1}\left(\sigma, \boldsymbol{\theta}, p, \boldsymbol{\theta}^{\prime}\right)=T_{3}^{1}\left[Z_{3}(\mathbf{s}) \delta(\sigma-\mathbf{s} \cdot \boldsymbol{\theta})\right]\left(p, \boldsymbol{\theta}^{\prime}\right)$.

Proof. By replacing $Y_{3}(\mathbf{s})$ in Eq. (3.6) by $Z_{3}(\mathbf{s}) \delta(\sigma-\mathbf{s} \cdot \boldsymbol{\theta})$ and using Lemma 3.1,

$$
T_{3}^{1}\left[X_{3} Z_{3}\right](\sigma, \boldsymbol{\theta})=\int X_{3}(\mathbf{s}) Z_{3}(\mathbf{s}) \delta(\sigma-\mathbf{s} \cdot \boldsymbol{\theta}) d \mathbf{s}=\Psi_{1}^{3}\left[\int X_{1, \boldsymbol{\theta}^{\prime}}(p) \widehat{Z}_{1}\left(\sigma, \boldsymbol{\theta}, p, \boldsymbol{\theta}^{\prime}\right) d p\right](\sigma, \boldsymbol{\theta})
$$

REMARK 3.1. Note that $\widehat{Z}_{1}\left(\sigma, \boldsymbol{\theta}, p, \boldsymbol{\theta}^{\prime}\right)$ is a function of a double projection on two separate direction vectors. This is related to the fact that the $T_{3}^{1}$ of a product of two RFs cannot be expressed in terms of the $T_{3}^{1} \mathrm{~s}$ of the individual functions evaluated for the same direction vector and projection. In fact, the relation below follows directly from the definition of the $T_{3}^{1}$ :

$$
T_{3}^{1}\left[X_{3} \mathbf{Z}_{3}\right](\sigma, \boldsymbol{\theta})=\int d p \widehat{X}_{1, \boldsymbol{\theta}}(\sigma=\mathbf{s} \cdot \boldsymbol{\theta}, \boldsymbol{\theta}) \widehat{Z}_{1, \boldsymbol{\theta}^{\prime}}\left(p=\mathbf{s} \cdot \boldsymbol{\theta}^{\prime}, \boldsymbol{\theta}^{\prime}\right) \delta\left(\mathbf{s}-\mathbf{s}^{\prime}\right)
$$

Finally, in view of Eq. (2.17) Plancherel's formula leads to an expression relating products of derivatives in the three- and the one-dimensional spaces,

$$
T_{3}^{1}\left[\sum_{i=1}^{3} \frac{\partial X_{3}}{\partial s_{i}} \frac{\partial Z_{3}}{\partial s_{i}}\right](\sigma, \boldsymbol{\theta})=\Psi_{1}^{3}\left[\int d p \frac{\partial X_{1, \boldsymbol{\theta}^{\prime}}(p)}{\partial p} \frac{\partial \widehat{Z}_{1}\left(\sigma, \boldsymbol{\theta}, p, \boldsymbol{\theta}^{\prime}\right)}{\partial p}\right]
$$

where the $\Psi_{1}^{3}$ is considered with respect to $\boldsymbol{\theta}^{\prime}$. 
4. Series expansions of space transformations. In several applications approximations based on series expansions of STs would be useful.

Proposition 4.1. The $T_{3}^{1}$ operator of the product of any two RFs, $X_{3}(\mathbf{s})$ and $Y_{3}(\mathbf{s})$, in $\mathbf{R}^{3}$ that admit individually the ST representation is given in terms of the series expansion

$$
\int \widehat{X}_{1 . \boldsymbol{\theta}}(\sigma) Y_{1 . \boldsymbol{\theta}}(\sigma) d \sigma=\sum_{k=0}^{\infty} \widehat{X}_{1 . \boldsymbol{\theta}}^{(k)}(\alpha) \int \frac{(\sigma-\alpha)^{k}}{k !} Y_{1, \boldsymbol{\theta}}(\sigma) d \sigma
$$

where $\widehat{X}_{1, \boldsymbol{\theta}}^{(k)}(\alpha)=\left.\frac{d^{k}}{d \sigma^{k}} \widehat{X}_{1, \boldsymbol{\theta}}(\sigma)\right|_{\sigma=\alpha}$. Equation (4.1) is exact, assuming that the Taylor expansion of $\widehat{X}_{1, \boldsymbol{\theta}}(\sigma)$ converges to $\widehat{X}_{1, \boldsymbol{\theta}}(\sigma)$.

Proof. By using Plancherel's formula (3.1) the $T_{3}^{1}$ of the product $X_{3}(\mathbf{s}) Y_{3}(\mathbf{s})$ can be written as

$$
\begin{aligned}
T_{3}^{1}\left[X_{3} Y_{3}\right](\sigma, \boldsymbol{\theta}) & =\int X_{3}(\mathbf{s}) Y_{3}(\mathbf{s}) \delta(\sigma-\mathbf{s} \cdot \boldsymbol{\theta}) d \mathbf{s} \\
& =\Psi_{1}^{3} \int\left[\int X_{3}(\mathbf{s}) \delta(\sigma-\mathbf{s} \cdot \boldsymbol{\theta}) \delta(p-\mathbf{s} \cdot \varphi) d \mathbf{s}\right] Y_{1 \cdot \varphi}(p) d p
\end{aligned}
$$

By integrating over $p$ at the left-hand side, that is, $T_{3}^{1}\left[X_{3} Y_{3}\right](\sigma, \boldsymbol{\theta})=$ $\Psi_{1}^{3} \int X_{3}(\mathbf{s}) \delta(\sigma-\mathbf{s} \cdot \boldsymbol{\theta}) Y_{1 . \varphi}(\mathbf{s} \cdot \varphi) d \mathbf{s}$, and then expanding $Y_{1 . \varphi}(\mathbf{s} \cdot \varphi)$ around $\alpha$, Eq. (4.2) leads to

$$
T_{3}^{1}\left[X_{3} Y_{3}\right](\sigma, \boldsymbol{\theta})=\sum_{k=0}^{\infty} \frac{1}{k !} \Psi_{1}^{3}\left\{\frac{d^{k} Y_{1, \varphi}(\alpha)}{d(\mathbf{s} \cdot \varphi)^{k}} T_{3}^{1}\left[(\mathbf{s} \cdot \varphi-\alpha)^{k} X_{3}\right](p, \boldsymbol{\theta})\right\} .
$$

By considering the integral on the right-hand side of Eq. (3.1) and taking the Taylor expansion of $\widehat{X}_{1 . \theta}(\sigma)$ around the point $\alpha$ one gets Eq. (4.1) above. This completes the proof.

REMARK 4.1. An interesting point to be noted regarding Taylor expansions of ST is that a meaningful expansion of $\widehat{X}_{1 . \theta}(\sigma)$ must contain more higher-order terms than a local expansion of $X_{3}(\mathbf{s})$, because $T_{3}^{1}$ assigns to each value of $\sigma$ the integral of $X_{3}(\mathbf{s})$ over a planar domain of points $\mathbf{s} \in \mathbf{R}^{3}$.

\section{The space transformation approach to stochastic partial differential} equations. As far as stochastic PDE with space-dependent coefficients are concerned, the ST approach consists in reducing the original multi-dimensional problem to a onedimensional problem. The solutions derived for the simpler one-dimensional problem can then be used to obtain solutions of the multi-dimensional problem by means of the inverse ST. Here we will focus on the $T_{n}^{1}$ transform. However, similar results can be obtained by means of the $\Psi_{n}^{1}$ transform.

Before applying the ST approach to stochastic PDE representing realistic porous media hydrodynamics, it may be instructive to try it first in a test problem. Indeed, the ST approach will be used here to derive solutions of the PDE in $\mathbf{R}^{2}$,

$$
\sum_{j=1}^{2}\left[\frac{\partial H_{2}(\mathbf{s})}{\partial s_{j}}+2 s_{j} H_{2}(\mathbf{s})\right]=0
$$


subject to the boundary condition $H_{2}(\mathbf{s}=0)=1$. The result will then be compared with the exact solution

$$
H_{2}(\mathbf{s})=\exp \left[-\mathbf{s}^{2}+\alpha\left(s_{1}-s_{2}\right)\right]
$$

where $\alpha$ is an arbitrary real constant. The solution (5.2) was obtained by the method of separation of variables (SoV; e.g., [1]). If $\alpha=0$, one recovers the isotropic solution

$$
H_{2}(\mathbf{s})=\exp \left(-\mathbf{s}^{2}\right)
$$

the $T_{2}^{1}$ of which is

$$
\widehat{H}_{1, \boldsymbol{\theta}}(p)=\sqrt{\pi} \exp \left(-p^{2}\right) .
$$

In this section the terms isotropic and anisotropic solution are used in reference to the spatial dependence of the solution. The isotropic solution depends entirely on the Euclidean norm of the position vector, and it has no directional dependence.

The application of the $T_{2}^{1}$ requires a coordinate system where the variables allow a straightforward integration of the delta function. This new coordinate system involves an orthogonal rotation of the axes in the two-dimensional plane. The transformation matrix is given by

$$
\mathbf{A}_{2}=\left[\begin{array}{cc}
\theta_{1} & \theta_{2} \\
-\theta_{2} & \theta_{1}
\end{array}\right]
$$

such that the new coordinates are $\mathbf{u}=\mathbf{A}_{2} \mathbf{z}$. The inverse matrix $\mathbf{A}_{2}^{-1}$ is given by

$$
\mathbf{A}_{2}^{-1}=\left[\begin{array}{cc}
\theta_{1} & -\theta_{2} \\
\theta_{2} & \theta_{1}
\end{array}\right]
$$

such that $\mathbf{z}=\mathbf{A}_{2}^{-1} \mathbf{u}=\mathbf{A}_{2}^{\mathrm{T}} \mathbf{u}$. The function $H_{2}(\mathbf{s})$ in the new coordinate system is denoted by $\mathbf{H}_{\boldsymbol{\theta}}(\mathbf{u})$, and the $T_{2}^{1}$ transforms Eq. (5.1) to the integro-differential equation

$$
\int d u_{2}\left\{\frac{\partial H_{\boldsymbol{\theta}}\left(p, u_{2}\right)}{\partial p}+2\left[p+u_{2} d(\boldsymbol{\theta})\right] H_{\boldsymbol{\theta}}\left(p, u_{2}\right)\right\}=0
$$

where $\boldsymbol{\theta}=\left(\theta_{1}, \theta_{2}\right)=(\cos \phi, \sin \phi), \phi$ being the rotation angle, and

$$
d(\boldsymbol{\theta})=\frac{\theta_{1}-\theta_{2}}{\theta_{1}+\theta_{2}}=\frac{\cos \varphi-\sin \phi}{\cos \phi+\sin \phi} .
$$

Note that only the derivative with respect to the projection on $\boldsymbol{\theta}$ appears in Eq. (5.7), and the generalized ST is a function of $H_{\boldsymbol{\theta}}\left(u_{1}, u_{2}\right)$, i.e.,

$$
\widehat{H}_{1, \boldsymbol{\theta}, \mathbf{z}}(p)=\delta\left(p-u_{1}\right) H_{\boldsymbol{\theta}}\left(u_{1}, u_{2}\right)
$$

The ordinary ST is then by definition a functional of $H_{\boldsymbol{\theta}}\left(u_{1}, u_{2}\right)$ given by

$$
\widehat{H}_{1 . \boldsymbol{\theta}}(p)=\int d u_{2} H_{\boldsymbol{\theta}}\left(p, u_{2}\right)
$$


Case I. Equation (5.7) will be solved by using the variable separability assumption of the standard SoV method which yields the exact solution (5.2), and then the ST result will be compared with the exact solution.

Indeed, it is assumed that the head function can be decomposed into two parametrized integrable functions $f_{\boldsymbol{\theta}}\left(u_{1}\right)$ and $g_{\boldsymbol{\theta}}\left(u_{2}\right)$, each of which depends on a projection of the position vector onto one of the basis axes of the new system, i.e.,

$$
H_{\boldsymbol{\theta}}\left(u_{1}, u_{2}\right)=f_{\boldsymbol{\theta}}\left(u_{1}\right) g_{\boldsymbol{\theta}}\left(u_{2}\right)
$$

The meaning of Eq. (5.10) is that the two-dimensional solution at a point $\mathbf{z}=\left(z_{1}, z_{2}\right) \in$ $\mathbf{R}^{2}$ is known if the values of $f_{\boldsymbol{\theta}}\left(u_{1}\right)$ and $g_{\boldsymbol{\theta}}\left(u_{2}\right)$ are known at the point $\mathbf{u}$ which is the map of point $\mathbf{z}$ under the orthogonal transformation (5.5). This in turn leads to the decomposition,

$$
\widehat{H}_{1, \boldsymbol{\theta}, \mathbf{z}}(p)=\delta\left(p-u_{1}\right) f_{\boldsymbol{\theta}}\left(u_{1}\right) g_{\boldsymbol{\theta}}\left(u_{2}\right) .
$$

The Jacobian of the orthogonal transformation (5.5) is equal to one and, therefore, there is no scaling factor for the integration in the rotated frame, i.e., $\int d \mathbf{z}=\int d \mathbf{u}$ (the integration is assumed to extend over an infinite region). In view of this fact, Eq. (5.7) becomes

$$
\int d u_{2}\left\{\frac{\partial f_{\boldsymbol{\theta}}(p)}{\partial p} g_{\boldsymbol{\theta}}\left(u_{2}\right)+2\left[p+u_{2} d(\boldsymbol{\theta})\right] f_{\boldsymbol{\theta}}(p) g_{\boldsymbol{\theta}}\left(u_{2}\right)\right\}=0 .
$$

At this point we can consider two possibilities regarding the function $g_{\boldsymbol{\theta}}\left(u_{2}\right)$.

(IA). Consider first the special case in which the function $g_{\boldsymbol{\theta}}\left(u_{2}\right)$ has even parity, i.e., $g_{\boldsymbol{\theta}}\left(-u_{2}\right)=g_{\boldsymbol{\theta}}\left(u_{2}\right)$.

In this case, the term in the integrand that has an explicit directional dependence through $d(\boldsymbol{\theta})$ integrates to zero and Eq. (5.12) is reduced to the considerably simpler ordinary differential equation (ODE)

$$
\frac{\partial f_{\boldsymbol{\theta}}(p)}{\partial p}+2 p f_{\boldsymbol{\theta}}(p)=0
$$

Equation (5.13) admits the solution $f_{\boldsymbol{\theta}}(p)=C \exp \left(-p^{2}\right)$, which leads to the isotropic ST

$$
\begin{aligned}
\widehat{H}_{1 . \boldsymbol{\theta}}(p) & =\int d \mathbf{z} \widehat{H}_{1, \boldsymbol{\theta} . \mathbf{z}}(p)=\int d \mathbf{u} \delta\left(p-u_{1}\right) f_{\boldsymbol{\theta}}\left(u_{1}\right) g_{\boldsymbol{\theta}}\left(u_{2}\right)=f_{\boldsymbol{\theta}}(p) \int d u_{2} g_{\boldsymbol{\theta}}\left(u_{2}\right) \\
& =C^{\prime} \exp \left(-p^{2}\right) .
\end{aligned}
$$

When $C^{\prime}=\sqrt{\pi}$, this is identical to the ST (5.4) of the isotropic case. Since the ST of a function identifies it uniquely, Eq. (5.14) implies that the ST method generates the isotropic solution of Eq. (5.1). This solution can be recovered directly by means of the two-dimensional inversion formula; see Eq. (1.6) for $n=2$.

REMARK 5.1. As follows from Eq. (5.14) the determination of the two-dimensional solution is possible on the basis only of the function $f_{\boldsymbol{\theta}}\left(u_{1}\right)$, since the information carried by $g_{\boldsymbol{\theta}}\left(u_{2}\right)$ is integrated to a mere multiplicative constant.

(IB). Consider now the more general case in which the even parity assumption is not valid and, hence, $\int_{-\infty}^{\infty} d u_{2} u_{2} g_{\boldsymbol{\theta}}\left(u_{2}\right) \neq 0$. 
Now Eq. (5.12) can be recast into the ODE

$$
\frac{\partial f_{\boldsymbol{\theta}}(p)}{\partial p}+2[p+v(\boldsymbol{\theta}) d(\boldsymbol{\theta})] f_{\boldsymbol{\theta}}(p)=0
$$

where

$$
v(\boldsymbol{\theta})=\frac{\int d u_{2} u_{2} g_{\boldsymbol{\theta}}\left(u_{2}\right)}{\int d u_{2} g_{\boldsymbol{\theta}}\left(u_{2}\right)} .
$$

Equation (5.15) admits a general solution of the form

$$
f_{\boldsymbol{\theta}}(p)=C_{1} \exp \left[-p^{2}-2 v(\boldsymbol{\theta}) d(\boldsymbol{\theta}) p\right]
$$

where an admissible function $v(\boldsymbol{\theta})$ must be determined, in the sense that it satisfies the original PDE (5.1).

The ODE (5.15) does not determine the function $g_{\boldsymbol{\theta}}\left(u_{2}\right)$, because it does not include derivatives with respect to the variable $u_{2}$. The function $g_{\boldsymbol{\theta}}\left(u_{2}\right)$ must be determined from the initial PDE (5.1). Indeed, after the application of the coordinate transformation given earlier, Eq. (5.1) becomes

$$
\frac{\partial f_{\boldsymbol{\theta}}\left(u_{1}\right)}{\partial u_{1}}+2 u_{1}+d(\boldsymbol{\theta})\left[\frac{\partial g_{\boldsymbol{\theta}}\left(u_{2}\right)}{\partial u_{2}}+2 u_{2} g_{\boldsymbol{\theta}}\left(u_{2}\right)\right]=0
$$

The solution for $g_{\boldsymbol{\theta}}\left(u_{2}\right)$ is determined by substituting the solution for $f_{\boldsymbol{\theta}}\left(u_{1}\right)$ derived from Eq. (5.17) into Eq. (5.18),

$$
g_{\boldsymbol{\theta}}\left(u_{2}\right)=C_{2} \exp \left[-u_{2}^{2}+2 v(\boldsymbol{\theta}) u_{2}\right]
$$

It is a simple matter to show that the $g_{\boldsymbol{\theta}}\left(u_{2}\right)$ of Eq. (5.19) satisfies the constitutive equation for $v(\boldsymbol{\theta})$, Eq. (5.16). The two-dimensional solution is now given directly in the rotated coordinate system as

$$
H_{2}(\mathbf{z})=C_{0} \exp \left[-u_{1}^{2}-u_{2}^{2}-2 v(\boldsymbol{\theta}) d(\boldsymbol{\theta}) u_{1}+2 v(\boldsymbol{\theta}) u_{2}\right]
$$

where $C_{0}=C_{1} C_{2}$. The inverse coordinate transformation defined by Eq. (5.6) should yield a function independent of the angle of rotation $\phi$. This natural constraint permits the determination of the directional function $v(\boldsymbol{\theta})$ in the exponent, $v(\boldsymbol{\theta})=\alpha(\cos \phi+\sin \phi)$, where $\alpha$ is an arbitrary real number. Thus, the final form of the ST solution is

$$
H_{2}(\mathbf{z})=C_{0} \exp \left[-z_{1}^{2}-z_{2}^{2}+\alpha\left(z_{1}-z_{2}\right)\right]=C_{0} \exp \left[-\mathbf{z}^{2}+\alpha\left(z_{1}-z_{2}\right)\right],
$$

which is in perfect agreement with the exact solution (5.2) derived by the standard SoV method.

Case II. In an attempt to show that ST is a more general approach than SoV, we will now study Eq. (5.7) without making the SoV assumption (5.11). 
It is assumed that the $H_{\boldsymbol{\theta}}\left(u_{1}, u_{2}\right)$ is a continuous function that is bounded over the flow domain, such that the integral of Eq. (5.9) exists for all $p \in \mathbf{R}^{1}$. Then the mean-value theorem guarantees the existence of a bounded real function $\tilde{v}(\boldsymbol{\theta})$ such that

$$
\int d u_{2} u_{2} H_{\boldsymbol{\theta}}\left(p, u_{2}\right)=\tilde{v}(\boldsymbol{\theta}) \int d u_{2} H_{\boldsymbol{\theta}}\left(p, u_{2}\right)=\tilde{v}(\boldsymbol{\theta}) \widehat{H}_{1 . \boldsymbol{\theta}}(p) .
$$

In light of Eqs. (5.9) and (5.22), Eq. (5.7) reduces to the ODE

$$
\frac{\partial \widehat{H}_{1 . \boldsymbol{\theta}}(p)}{\partial p}+2[p+\tilde{v}(\boldsymbol{\theta}) d(\boldsymbol{\theta})] \widehat{H}_{1 . \boldsymbol{\theta}}(p)=0 .
$$

The above equation is easily solved by standard ODE methods and yields the following ST field as a functional of $\tilde{v}(\boldsymbol{\theta})$,

$$
\widehat{H}_{1 . \boldsymbol{\theta}}(p)=\widehat{H}_{1 . \boldsymbol{\theta}}(0) \exp \left[-p^{2}-\tilde{v}(\boldsymbol{\theta}) d(\boldsymbol{\theta}) p\right] .
$$

In general, the functions $\tilde{v}(\boldsymbol{\theta})$ and $\widehat{H}_{1, \boldsymbol{\theta}}(0)$ must be determined self-consistently so that Eq. (5.1) is satisfied. Note that if we use the separability assumption (5.11), Eq. (5.23) reduces to Eq. (5.15). Moreover, if $\tilde{v}(\boldsymbol{\theta})=0$ and $\widehat{H}_{1, \boldsymbol{\theta}}(0)=$ const., Eq. (5.24) gives the ST of the isotropic solution, Eq. (5.4).

By way of a summary, we have shown that (a) under the assumption of variable separability, the ST method and the standard SoV method generate the same solution for the test problem above; (b) the ST approach does not need the SoV assumption in order to obtain the isotropic solution; (c) if the assumption of variable separability is not used, the ST of the anisotropic solution can still be determined partially.

REMARK 5.2. ST can be calculated for different directional functions and inverted by application of inverse ST techniques. It should be verified that the resulting functions satisfy the initial PDE (5.1). This is not surprising since the original PDE includes partial derivatives of the unknown function with respect to two independent space variables. On the other hand, the resulting one-dimensional equation involves derivatives with respect to a single variable. If the PDE solution is not spatially isotropic it cannot be completely determined from the one-dimensional equation per se. Hence, the sensitivity of the original PDE on variations of the directional function is a question that merits further study.

6. Groundwater flow in three-dimensional porous formations. Consider the steady-state, three-dimensional groundwater flow in a porous formation governed by the PDE

$$
\sum_{j=1}^{3}\left[\frac{\partial^{2} H_{3}(\mathbf{s})}{\partial s_{j}^{2}}+w_{j}(\mathbf{s}) \frac{\partial H_{3}(\mathbf{s})}{\partial s_{j}}\right]=0,
$$

where $w_{j}(\mathbf{s})=\frac{\partial \ln K_{3}(\mathbf{s})}{\partial s_{j}} ; K_{3}(\mathbf{s})$ is the scalar (and hence isotropic) hydraulic conductivity, $H_{3}(\mathbf{s})$ is the hydraulic head, and $w_{j}(\mathbf{s})$ are the random Cartesian components of the log-conductivity gradient. The log-conductivity $\ln K_{3}(\mathbf{s})$ is assumed to be an RF with known spatial correlation structure. Although in this paper hydraulic conductivity will 
be viewed as an isotropic tensor, i.e., as a scalar, the analysis can be extended to the case of an anisotropic (tensor) hydraulic conductivity, as is shown below. For the case of a scalar hydraulic conductivity, a distinction will be made between isotropic and anisotropic spatial dependence. As regards boundary conditions (BC), we will first consider the case of an infinite flow domain, where Eq. (6.1) is conditioned by a set of point-like BC to be specified in the flow domain. This represents a reasonable scenario in practice. Then, we will use the ST analysis to study the case of Neumann BC on a spherical domain.

An application of the $T_{3}^{1}$ to Eq. (6.1) involves the following transformations $(j=1,2$, and 3 ), which are valid assuming that the boundary terms vanish:

$$
\begin{gathered}
T_{3}^{1}\left[\frac{\partial H_{3}(\mathbf{s})}{\partial s_{j}}\right](\sigma, \boldsymbol{\theta})=\theta_{j} \frac{\partial \widehat{H}_{1, \boldsymbol{\theta}}(\sigma)}{\partial \sigma}, \\
T_{3}^{1}\left[\frac{\partial^{2} H_{3}(\mathbf{s})}{\partial s_{j}^{2}}\right](\sigma, \boldsymbol{\theta})=\theta_{j}^{2} \frac{\partial \widehat{H}_{1, \boldsymbol{\theta}}^{2}(\sigma)}{\partial \sigma^{2}},
\end{gathered}
$$

and

$$
\widehat{H}_{1, \boldsymbol{\theta}}(\sigma)=\int d \mathbf{z} \widehat{H}_{1, \boldsymbol{\theta}, \mathbf{z}}(\sigma)
$$

where $\widehat{H}_{1, \boldsymbol{\theta}, \mathbf{z}}(\sigma)$ is the generalized ST of the head. In light of the above, Eq. (6.1) leads to an expression involving the spatial integration of an ODE, viz.,

$$
\int_{D} d \mathbf{z}\left[\frac{\partial^{2} \widehat{H}_{1, \boldsymbol{\theta}, \mathbf{z}}(\sigma)}{\partial \sigma^{2}}+\kappa_{\boldsymbol{\theta}}(\mathbf{z}) \frac{\partial \widehat{H}_{1, \boldsymbol{\theta}, \mathbf{z}}(\sigma)}{\partial \sigma}\right]=0
$$

where the projected gradient of the log-conductivity

$$
\kappa_{\boldsymbol{\theta}}(\mathbf{z})=\sum_{j=1}^{3} \theta_{j} w_{j}(\mathbf{z})
$$

is a scalar RF with a value at point $\mathbf{z}$ equal to the projection of the log-conductivity gradient onto the sampling line specified by the direction vector $\boldsymbol{\theta}$. The $\kappa_{\boldsymbol{\theta}}(\mathbf{z})$ plays an important role in the ST analysis, for it carries a significant amount of the information which is transferred from the three-dimensional space to the one-dimensional space.

At this point it is convenient to introduce an orthogonal coordinate transformation matrix

$$
\mathbf{A}_{3}=\left[\begin{array}{ccc}
\theta_{1} & \theta_{2} & \theta_{3} \\
-\theta_{1} \theta_{2} / q & q & -\theta_{2} \theta_{3} / q \\
-\theta_{3} / q & 0 & \theta_{1} / q
\end{array}\right]
$$

The matrix $\mathbf{A}_{3}$ applies a rotation by a solid angle $\boldsymbol{\theta}$ so that

$$
\mathbf{u}^{\mathrm{T}}=\mathbf{A}_{3} \cdot \mathbf{z}
$$

The rotation brings one of the axes of the new system on the line of the direction vector of the ST. The parameters of the rotation matrix are $\boldsymbol{\theta}=\left(\theta_{1}, \theta_{2}, \theta_{3}\right)=(\sin \chi \cos \phi, \sin \chi \sin \phi$, 
$\cos \chi), \chi$ and $\phi$ being respectively the polar and the azimuthal angle of the unit direction vector $\boldsymbol{\theta}$, and $q=\sqrt{\theta_{1}^{2}+\theta_{3}^{2}}$. In the new coordinate system, identified by the vector parameter $\boldsymbol{\theta}$, the position variables $\left(s_{1}, s_{2}, s_{3}\right)$ and $\left(z_{1}, z_{2}, z_{3}\right)$ are replaced by the new variables $\left(u_{1}, u_{2}, u_{3}\right)$. In this system the projection length of a vector $\mathbf{z}$ (or $\mathbf{s}$ ) becomes an independent space variable, i.e.,

$$
\sum_{i=1}^{3} z_{i} \theta_{i}=u_{1}
$$

and the head becomes a function of the vector variable $\mathbf{u}$, parametrized by the direction vector $\boldsymbol{\theta}$.

The solution for the generalized ST head, which is consistent with the definition of Eq. (2.2), is of the form

$$
\widehat{H}_{1, \boldsymbol{\theta}, \mathbf{z}}(\sigma)=\delta\left(\sigma-u_{1}\right) H_{\boldsymbol{\theta}}(\mathbf{u}) .
$$

Note that $H_{\theta}(\mathbf{u})$ is the value of the three-dimensional head potential at the point $\mathbf{z}$ of the flow domain expressed in the rotated coordinate frame. It is also convenient to define the one-dimensional generalized head slope as the slope of the generalized ST of the head with respect to the projection length $\sigma$,

$$
\frac{\partial \widehat{H}_{1, \boldsymbol{\theta} . \mathbf{z}}(\sigma)}{\partial \sigma}=-\frac{\partial \delta\left(\sigma-u_{1}\right)}{\partial u_{1}} H_{\boldsymbol{\theta}}(\mathbf{u})=\delta\left(\sigma-u_{1}\right) \frac{\partial H_{\boldsymbol{\theta}}(\mathbf{u})}{\partial u_{1}}
$$

and, by repeated differentiation,

$$
\frac{\partial^{2} \hat{H}_{1, \boldsymbol{\theta}, \mathbf{z}}(\sigma)}{\partial \sigma^{2}}=\delta\left(\sigma-u_{1}\right) \frac{\partial^{2} H_{\boldsymbol{\theta}}(\mathbf{u})}{\partial u_{1}^{2}} .
$$

Finally, in order to simplify notation we define on $\mathbf{R}^{3}$ the first and second negative derivatives of the head with respect to the projection length

$$
J_{\boldsymbol{\theta}}\left(\sigma, \mathbf{u}^{\prime}\right)=-\frac{\partial H_{\boldsymbol{\theta}}\left(\sigma, \mathbf{u}^{\prime}\right)}{\partial \sigma}
$$

and

$$
\frac{\partial J_{\boldsymbol{\theta}}\left(\sigma, \mathbf{u}^{\prime}\right)}{\partial \sigma}=-\frac{\partial H_{\boldsymbol{\theta}}^{2}\left(\sigma, \mathbf{u}^{\prime}\right)}{\partial \sigma^{2}},
$$

where $\mathbf{u}^{\prime}=\left(u_{2}, u_{3}\right)$. Equation (6.13) defines the hydraulic head gradient along the direction vector.

In order to calculate the one-dimensional head slope, i.e., the derivative with respect to $\sigma$ of the ST of the head, the integration over $u_{1}$ is readily performed by virtue of the delta function. Since the differentiation with respect to the projection length and the integration over the space variables in the orthogonal directions are interchangeable operations, the following expression is obtained for the one-dimensional negative head slope:

$$
\widehat{J}_{1 . \boldsymbol{\theta}}(\sigma) \equiv-\frac{\partial \widehat{H}_{1 . \boldsymbol{\theta}}(\sigma)}{\partial \sigma}=-\int_{D} d \mathbf{z} \frac{\partial \widehat{H}_{1 . \boldsymbol{\theta} . \mathbf{z}}(\sigma)}{\partial \sigma}=\int d \mathbf{u}^{\prime} J_{\boldsymbol{\theta}}\left(\sigma, \mathbf{u}^{\prime}\right)
$$


where the double integral $\int d u_{2} \int d u_{3}$ is understood as $\int d \mathbf{u}^{\prime}$. In view of the analysis above, Eq. (6.5) can now be written in the language of the new formalism. Indeed, after the integration over the delta function has been performed, the following result is obtained:

$$
\int d \mathbf{u}^{\prime}\left[\frac{\partial J_{\boldsymbol{\theta}}\left(\sigma, \mathbf{u}^{\prime}\right)}{\partial \sigma}+\kappa_{\boldsymbol{\theta}}\left(\sigma, \mathbf{u}^{\prime}\right) J_{\boldsymbol{\theta}}\left(\sigma, \mathbf{u}^{\prime}\right)\right]=0 .
$$

At this point, we have transformed the three-dimensional PDE (6.1) to the onedimensional integro-differential Eq. (6.16). After Eq. (6.16) has been solved for $J_{\boldsymbol{\theta}}\left(\sigma, \mathbf{u}^{\prime}\right)$, the $\widehat{H}_{1, \boldsymbol{\theta}}(\sigma)$ can be found from Eq. (6.15) and then the required three-dimensional solution $H_{3}(\mathbf{s})$ can be obtained by applying $T_{1}^{3}$.

To proceed further with the analysis we define the weighted projected gradient of the $\log$-conductivity,

$$
\tilde{\kappa}_{\boldsymbol{\theta}}(\sigma)=\frac{\int d \mathbf{u}^{\prime} J_{\boldsymbol{\theta}}\left(\sigma, \mathbf{u}^{\prime}\right) \kappa_{\boldsymbol{\theta}}\left(\sigma, \mathbf{u}^{\prime}\right)}{\int d \mathbf{u}^{\prime} J_{\boldsymbol{\theta}}\left(\sigma, \mathbf{u}^{\prime}\right)},
$$

so that Eq. (6.16) is reduced to the first-order ODE

$$
\frac{\partial \widehat{J}_{1, \boldsymbol{\theta}}(\sigma)}{\partial \sigma}+\tilde{\kappa}_{\boldsymbol{\theta}}(\sigma) \widehat{J}_{1, \boldsymbol{\theta}}(\sigma)=0 .
$$

The problem of solving the initial stochastic PDE (6.1) has now been reduced to the problem of solving the ODE (6.18) with respect to $\widehat{J}_{1, \boldsymbol{\theta}}(\sigma)$. The solution is

$$
\widehat{J}_{1, \boldsymbol{\theta}}(\sigma)=\widehat{J}_{1, \boldsymbol{\theta}}(0) \exp \left[-\int_{0}^{\sigma} d u_{1} \tilde{\kappa}_{\boldsymbol{\theta}}\left(u_{1}\right)\right] \text {. }
$$

Equations (6.17) and (6.19) establish the full solution of the one-dimensional problem. After the one-dimensional $\widehat{J}_{i, \theta}(\sigma)$ has been calculated, the three-dimensional head solution can be determined by application of the inverse ST,

$$
\begin{aligned}
H_{3}(\mathbf{s}) & =T_{1}^{3}\left[\widehat{H}_{1, \boldsymbol{\theta}}(\sigma)\right]=-\frac{1}{2(2 \pi)^{2}} \int_{S_{3}} \frac{\partial^{2} \widehat{H}_{1, \boldsymbol{\theta}}(\sigma)}{\partial \sigma^{2}} d \boldsymbol{\theta} \\
& =\frac{1}{2(2 \pi)^{2}} \int_{S_{3}} \frac{\partial \widehat{J}_{1, \boldsymbol{\theta}}(\sigma)}{\partial \sigma} d \boldsymbol{\theta}
\end{aligned}
$$

at $\sigma=\mathbf{s} \cdot \boldsymbol{\theta}$. Equation (6.19) and, hence, Eq. (6.21) contain two undetermined functions: the weighted projected gradient of the log-conductivity $\tilde{\kappa}_{\boldsymbol{\theta}}(\mathbf{u})$ and the directional distribution $\widehat{J}_{1, \theta}(0)$. These should be determined by means of the self-consistency rule [i.e., so that the three-dimensional head solution (6.21) satisfies the original PDE (6.1)] and the point-like BC (measurements). The family of the solutions of the one-dimensional problem is, in general, larger than the family of the solutions of the initial three-dimensional problem (this was already demonstrated in Sec. 5). The determination of $\widehat{J}_{1 . \boldsymbol{\theta}}(0)$ from the point-like $\mathrm{BC}$ is discussed in more detail in Sec. 8 below. The analysis above completes the proof of the following 
Proposition 6.1. The solution of the PDE (6.1) in the case of an infinite flow domain where Eq. (6.1) is conditioned by a set of point-like BC in the flow domain is given by Eq. (6.21), where $\widehat{J}_{1, \boldsymbol{\theta}}(\sigma)$ is the solution (6.19) of the ODE (6.18).

REMARK 6.1. By introducing the directional head generator

$$
m(\sigma ; \boldsymbol{\theta})=\tilde{\kappa}_{\boldsymbol{\theta}}(\sigma) \exp \left[-\int_{0}^{\sigma} d u_{1} \tilde{\kappa}_{\boldsymbol{\theta}}\left(u_{1}\right)\right]
$$

Eq. (6.21) can also be expressed in the following elegant form:

$$
H_{3}(\mathbf{s})=-\frac{1}{2(2 \pi)^{2}} \int d \boldsymbol{\theta} \widehat{J}_{1, \boldsymbol{\theta}}(0) m(\sigma, \boldsymbol{\theta}),
$$

at $\sigma=\mathbf{s} \cdot \boldsymbol{\theta}$.

REMARK 6.2. The analysis above can easily be extended to the case of an anisotropic hydraulic conductivity tensor with the following modifications. The projected gradient of the log-conductivity, i.e., of Eq. (6.6), now becomes

$$
\kappa_{\boldsymbol{\theta}}(\mathbf{z})=\frac{\sum_{j=1}^{3} \sum_{k=1}^{3} \theta_{j} \frac{\partial K_{j k}(\mathbf{z})}{\partial z_{j}}}{\sum_{j=1}^{3} \sum_{k=1}^{3} \theta_{j} \theta_{k} K_{j k}(\mathbf{z})}
$$

and the weighted projected gradient of the log-conductivity, i.e., Eq. (6.17) becomes

$$
\tilde{\kappa}_{\boldsymbol{\theta}}(\sigma)=\frac{T_{3}^{1}\left[\sum_{j=1}^{3} \theta_{j} D_{j}\left[\mathbf{K}_{3}(\mathbf{s}), \mathbf{Q}_{3}(\mathbf{s})\right]\left[\operatorname{det} \mathbf{K}_{3}(\mathbf{s})\right]^{-1} \kappa_{\boldsymbol{\theta}}(\mathbf{s})\right](\sigma, \boldsymbol{\theta})}{T_{3}^{1}\left[\sum_{j=1}^{3} \theta_{j} D_{j}\left[\mathbf{K}_{3}(\mathbf{s}), \mathbf{Q}_{3}(\mathbf{s})\right]\left[\operatorname{det} \mathbf{K}_{3}(\mathbf{s})\right]^{-1}\right](\sigma, \boldsymbol{\theta})},
$$

where $D_{j}\left[\mathbf{K}_{3}(\mathbf{s}), \mathbf{Q}_{3}(\mathbf{s})\right](j=1,2,3)$ are determinants of the matrices that result from the substitution of the $j$ th column in the matrix representing the hydraulic conductivity tensor with the specific discharge vector, e.g.,

$$
\begin{gathered}
D_{1}\left[\mathbf{K}_{3}(\mathbf{s}), \mathbf{Q}_{33}(\mathbf{s})\right]=\left|\begin{array}{lll}
Q_{3.1}(\mathbf{s}) & K_{12}(\mathbf{s}) & K_{13}(\mathbf{s}) \\
Q_{3.2}(\mathbf{s}) & K_{22}(\mathbf{s}) & K_{23}(\mathbf{s}) \\
Q_{3.3}(\mathbf{s}) & K_{32}(\mathbf{s}) & K_{33}(\mathbf{s})
\end{array}\right| \\
\mathbf{Q}_{3}(\mathbf{s})=\left[Q_{3.1}(\mathbf{s}), Q_{3.2}(\mathbf{s}), Q_{3.3}(\mathbf{s})\right]
\end{gathered}
$$

It may be instructive to consider some specific examples representing situations where straightforward analytic expressions for the solutions (6.21) or (6.23) can be established.

EXAMPLE 6.1. In certain cases explicit formulas can be derived for $\tilde{\kappa}_{\boldsymbol{\theta}}(u)$ in terms of the hydraulic conductivity field and its gradients. One such case is groundwater flow at a constant specific discharge vector $\mathbf{Q}_{3}\left(\mathbf{Q}_{3}\right.$ is assumed to be known by means of its components $Q_{3, i} ; i=1,2$, and 3). Darcy's law (e.g., [10]) relates $\mathbf{Q}_{3}$ with the gradient of the hydraulic head and the hydraulic conductivity of the porous medium,

$$
K_{3}(\mathbf{s}) \nabla H_{3}(\mathbf{s})=-\mathbf{Q}_{3} .
$$

This form of Darcy's law implies that the equipotentials are the planes $\mathbf{Q}_{3} \cdot \mathbf{s}=$ const. which, in turn, imposes certain restrictions on the functional form of the solution $H_{3}(\mathbf{s})$. An explicit formula for $\tilde{\kappa}_{\boldsymbol{\theta}}(u)$ is suggested by 
LEMMA 6.1. In the general case of a constant specific discharge vector $\mathbf{Q}_{3}$,

$$
\tilde{\kappa}_{\boldsymbol{\theta}}\left(u_{1}\right)=\frac{T_{3}^{1}\left[K_{3}^{-1}(\mathbf{s}) \kappa_{\boldsymbol{\theta}}(\mathbf{s})\right]\left(u_{1}, \boldsymbol{\theta}\right)}{T_{3}^{1}\left[K_{3}^{-1}(\mathbf{s})\right]\left(u_{1}, \boldsymbol{\theta}\right)} .
$$

Proof. Using the properties of the ST derivatives,

$$
\sum_{i=1}^{3} \theta_{i} T_{3}^{1}\left[\frac{\partial H_{3}(\mathbf{s})}{\partial s_{i}}\right]=\sum_{i=1}^{3} \theta_{i}^{2}\left[\frac{\partial \widehat{H}_{1, \boldsymbol{\theta}}(\sigma)}{\partial \sigma}\right]=\frac{\partial \widehat{H}_{1, \boldsymbol{\theta}}(\sigma)}{\partial \sigma}
$$

The one-dimensional head slope can be expressed in terms of the one-dimensional negative head slope or in terms of the denominator of the weighted projected gradient of the log-conductivity, i.e.,

$$
-\frac{\partial \widehat{H}_{1, \boldsymbol{\theta}}(\sigma)}{\partial \sigma}=\widehat{J}_{1, \boldsymbol{\theta}}(\sigma)=\int d \mathbf{u}^{\prime} J_{\boldsymbol{\theta}}\left(\sigma, \mathbf{u}^{\prime}\right)
$$

By using Darcy's law in the left-hand side of Eq. (6.29), the one-dimensional head slope equals

$$
\sum_{i=1}^{3} \theta_{i} T_{3}^{1}\left[\frac{\partial H_{3}(\mathbf{s})}{\partial s_{i}}\right]=-\sum_{i=1}^{3} \theta_{i} Q_{3, i} T_{3}^{1}\left[K_{3}^{-1}(\mathbf{s})\right] ;
$$

by virtue of Eq. (6.30) the denominator of the weighted projected gradient of the logconductivity becomes

$$
\int d \mathbf{u}^{\prime} J_{\boldsymbol{\theta}}\left(\sigma, \mathbf{u}^{\prime}\right)=\sum_{i=1}^{3} \theta_{i} Q_{3, i} T_{3}^{1}\left[K_{3}^{-1}(\mathbf{s})\right]
$$

The numerator of $\tilde{\kappa}_{\boldsymbol{\theta}}(\mathbf{u})$ in Eq. (6.17) can also be written as

$$
\int d \mathbf{u}^{\prime} J_{\boldsymbol{\theta}}\left(\sigma, \mathbf{u}^{\prime}\right) \kappa_{\boldsymbol{\theta}}\left(\sigma, \mathbf{u}^{\prime}\right)=\sum_{i} \theta_{i}^{2} \int d \mathbf{u}^{\prime} J_{\boldsymbol{\theta}}\left(\sigma, \mathbf{u}^{\prime}\right) \kappa_{\boldsymbol{\theta}}\left(\sigma, \mathbf{u}^{\prime}\right)
$$

Now notice that

$$
\begin{aligned}
\sum_{i=1}^{3} \theta_{i}^{2} \int d \mathbf{u}^{\prime} J_{\boldsymbol{\theta}}\left(\sigma, \mathbf{u}^{\prime}\right) \kappa_{\boldsymbol{\theta}}\left(\sigma, \mathbf{u}^{\prime}\right)=-\sum_{i=1}^{3} \theta_{i}^{2} \int d \mathbf{s} \frac{\partial H_{3}(\mathbf{s})}{\partial \sigma} \kappa_{\boldsymbol{\theta}}(\mathbf{s}) \delta(\sigma-\mathbf{s} \cdot \boldsymbol{\theta}) \\
\quad=-\sum_{i=1}^{3} \theta_{i}^{2} \int d \mathbf{s} \lim _{\varepsilon \rightarrow 0} \frac{H_{3}\left(\mathbf{s}+\mathbf{e}_{i} \frac{\varepsilon}{\theta_{i}}\right)-H_{3}(\mathbf{s})}{\varepsilon} \kappa_{\boldsymbol{\theta}}(\mathbf{s}) \delta(\sigma-\mathbf{s} \cdot \boldsymbol{\theta}) \\
=-\sum_{i=1}^{3} \theta_{i}^{2} \int d \mathbf{s} \lim _{\varepsilon^{\prime} \rightarrow 0} \frac{H_{3}\left(\mathbf{s}+\mathbf{e}_{i} \varepsilon^{\prime}\right)-H_{3}(\mathbf{s})}{\varepsilon^{\prime} \theta_{i}} \kappa_{\boldsymbol{\theta}}(\mathbf{s}) \delta(\sigma-\mathbf{s} \cdot \boldsymbol{\theta}) \\
=-\sum_{i=1}^{3} \theta_{i} T_{3}^{1}\left[\frac{\partial H_{3}(\mathbf{s})}{\partial s_{i}} \kappa_{\boldsymbol{\theta}}(\mathbf{s})\right]
\end{aligned}
$$


where the unit vectors in the three orthogonal directions are denoted by $\mathbf{e}_{i}, i=1,2,3$. Using Darcy's law,

$$
-\sum_{i=1}^{3} \theta_{i} T_{3}^{1}\left[\frac{\partial H_{3}(\mathbf{s})}{\partial s_{i}} \kappa_{\boldsymbol{\theta}}(\mathbf{s})\right]=\sum_{i=1}^{3} \theta_{i} Q_{3, i} T_{3}^{1}\left[K_{3}^{-1}(\mathbf{s}) \kappa_{\boldsymbol{\theta}}(\mathbf{s})\right] .
$$

The numerator of Eq. (6.17) follows simply from Eqs. (6.34) and (6.35), viz.

$$
\int d \mathbf{u}^{\prime} J_{\boldsymbol{\theta}}\left(\sigma, \mathbf{u}^{\prime}\right) \kappa_{\boldsymbol{\theta}}\left(\sigma, \mathbf{u}^{\prime}\right)=\sum_{i=1}^{3} \theta_{i} Q_{3 . i} T_{3}^{1}\left[K_{3}^{-1}(\mathbf{s}) \kappa_{\boldsymbol{\theta}}(\mathbf{s})\right]
$$

Finally, by inserting Eqs. (6.32) and (6.36) into Eq. (6.17) we obtain Eq. (6.28).

Equation (6.28) can be used together with Eqs. (6.19) and (6.21) to derive the analytical form of the solution $H_{3}(\mathbf{s})$. This completes Example 6.1.

EXAMPLE 6.2. Some interesting analytical solutions can be derived in the isotropic case. Assume that the hydraulic head is an isotropic RF, i.e., that $H_{3}$ (s) depends purely on the Euclidean norm $r=|\mathbf{s}|$ of the position vector measured from the origin: $H_{3}(\mathbf{s})=$ $H(r)$. Its ST transform is also an isotropic function that depends exclusively on the projection length $\sigma$, as is clear by the following:

$$
\widehat{H}_{1 . \boldsymbol{\theta}}(\sigma)=\int d \mathbf{s} \delta(\sigma-\mathbf{s} \cdot \boldsymbol{\theta}) H(r)=\int d \mathbf{u}^{\prime} H\left(\sqrt{\sigma^{2}+\mathbf{u}^{\prime 2}}\right) .
$$

A sufficient condition for an isotropic solution to exist is that the PDE (6.1) is invariant under interchange of any two space variables. That this condition is not also necessary is made obvious by the counterexample $\frac{\partial H_{33}(\mathbf{s})}{\partial s_{1}}+2 s_{1} H_{3}(\mathbf{s})=0$, that admits the isotropic solution $H_{3}(\mathbf{s})=\exp \left(-s^{2}\right)$. The condition for an isotropic solution is satisfied when the log-conductivity is an isotropic RF, i.e., when the following is true:

$$
\ln K_{3}(\mathbf{s})=\alpha(r)
$$

where $\alpha(r)$ is a uni-dimensional RF. Then, the projected gradient of the log-conductivity defined in Eq. (6.6) becomes

$$
\kappa_{\boldsymbol{\theta}}(\mathbf{z})=\boldsymbol{\theta} \cdot \mathbf{z} \frac{1}{r} \frac{\partial \alpha(r)}{\partial r}=u_{1} \beta(r)
$$

A specific situation that permits a decomposition of the projected gradient of the logconductivity will be considered, where the projected gradient of the log-conductivity is assumed to admit a factorial decomposition,

$$
\beta(r)=\beta^{\prime}\left(u_{1}\right) \beta^{\prime \prime}\left(\mathbf{u}^{\prime}\right) .
$$

The one-dimensional flow Eq. (6.16) now becomes

$$
\int d \mathbf{u}^{\prime}\left[\frac{\partial J_{\boldsymbol{\theta}}\left(\sigma, \mathbf{u}^{\prime}\right)}{\partial \sigma}+\sigma \beta^{\prime}\left(u_{1}\right) \beta^{\prime \prime}\left(\mathbf{u}^{\prime}\right) J_{\boldsymbol{\theta}}\left(\sigma, \mathbf{u}^{\prime}\right)\right]=0
$$


and by using Eq. (6.15) and the mean-value theorem,

$$
\int d \mathbf{u}^{\prime}\left[\beta^{\prime \prime}\left(\mathbf{u}^{\prime}\right) J_{\boldsymbol{\theta}}\left(\sigma, \mathbf{u}^{\prime}\right)\right]=\gamma(\boldsymbol{\theta}) \int d \mathbf{u}^{\prime} J_{\boldsymbol{\theta}}\left(\sigma, \mathbf{u}^{\prime}\right)=\gamma(\boldsymbol{\theta}) \widehat{J}_{1, \boldsymbol{\theta}}(\sigma),
$$

where $\gamma(\boldsymbol{\theta})$ is a directional function that depends in general on the direction vector. An isotropic solution requires that $\gamma(\boldsymbol{\theta})=\gamma_{0}$. The one-dimensional negative head slope solution of Eq. (6.41) follows easily,

$$
\widehat{J_{1 . \theta}}(\sigma)=J_{0} \exp \left[-\gamma_{0} \int_{0}^{\sigma} d u_{1} u_{1} \beta^{\prime}\left(u_{1}\right)\right] .
$$

For an isotropic solution the value of $\widehat{J}_{1, \boldsymbol{\theta}}(\sigma)$ at zero projection length must be a constant independent of the direction vector $\boldsymbol{\theta}$, i.e., $\widehat{J}_{1, \boldsymbol{\theta}}(0)=J_{(0)}$. The three-dimensional head can now be calculated by applying the inverse ST to Eq. (6.43) according to Eq. $(6.21)$

$$
H_{3}(\mathbf{s})=-\frac{\gamma_{0}}{2(2 \pi)^{2}} J_{0} \int d \boldsymbol{\theta}(\mathbf{s} \cdot \boldsymbol{\theta}) \beta^{\prime}(\mathbf{s} \cdot \boldsymbol{\theta}) \exp \left[-\gamma_{0} \int_{0}^{\sigma=\mathbf{s} \cdot \boldsymbol{\theta}} d u_{1} u_{1} \beta^{\prime}\left(u_{1}\right)\right] .
$$

This completes Example 6.2. Note that Eq. (6.40) is satisfied if $\beta(r)=\beta_{0} \exp \left(-r^{2}\right)$.

Finally, we will examine the flow Eq. (6.1) in the case of a specific boundary geometry.

Proposition 6.2. The solution of the PDE (6.1) in the case of the Neumann BC

$$
\boldsymbol{\eta} \cdot \nabla H_{3}\left(\mathbf{s}_{B}\right)=\phi_{3}\left(\mathbf{s}_{B}\right)
$$

at $\mathbf{s}_{B} \in B$, where $B$ is a spherical domain and $\boldsymbol{\eta}$ is a unit vector perpendicular to $B$, is given by Eq. (6.21) where now the one-dimensional solution is

$$
\widehat{J}_{1, \boldsymbol{\theta}}(\sigma)=-\phi_{3}\left(\mathbf{s}_{B}\right) \exp \left[-\int_{\sigma_{B}}^{\sigma} d \mathbf{u} \tilde{\kappa}_{\boldsymbol{\theta}}(\mathbf{u})\right] .
$$

Proof. By applying the ST $T_{3}^{1}$ to Eq. (6.45), and due to the spherical form of the boundary, we find

$$
-\widehat{J}_{1, \boldsymbol{\theta}}\left(\sigma_{B}\right)=\phi_{3}\left(\mathbf{s}_{B}\right)
$$

at $\sigma_{B}=\mathbf{s}_{B} \cdot \boldsymbol{\theta}$. In this case the solution $\widehat{J}_{1, \boldsymbol{\theta}}(\sigma)$ of the ODE (6.18) subject to Eq. (6.46) is found simply by replacing $\widehat{J}_{1, \boldsymbol{\theta}}(0)$ in Eq. (6.19) with $-\phi_{3}\left(\mathbf{s}_{B}\right)$. This gives Eq. (6.46) and completes the proof.

7. Analysis in terms of Taylor series expansions of the log-conductivity gradient. A solution of the steady-state three-dimensional flow governed by Eq. (6.1) will be attempted by direct expansion of the components of the log-conductivity gradient around the neighborhood of a point $\mathbf{s}_{0} \in D$, viz.

$$
w_{j}(\mathbf{s})=\left.\sum_{n=0}^{\infty} \frac{1}{n !}\left[\left(\mathbf{s}-\mathbf{s}_{0}\right) \cdot \nabla\right]^{n} w_{j}(\mathbf{s})\right|_{\mathbf{s}=\mathbf{s}_{0}} .
$$


The binomial expansion can be used to derive the following expression for the part of the Taylor series that displays an explicit $\mathbf{s}$ dependence:

$$
\left[\left(\mathbf{s}-\mathbf{s}_{0}\right) \cdot \nabla_{\mathbf{s}_{0}}\right]^{n}=\sum_{k=0}^{n} C_{n}^{k}\left(\mathbf{s} \cdot \nabla_{\mathbf{s}_{0}}\right)^{k}\left(\mathbf{s}_{0} \cdot \nabla_{\mathbf{s}_{0}}\right)^{n-k}
$$

where $C_{n}^{k}=\left(\begin{array}{l}n \\ k\end{array}\right)$, and the subscript $\mathbf{s}_{0}$ on the gradient operator implies that the latter operates on $w_{j}(\mathbf{s})$ and the result is then evaluated at the point $\mathbf{s}_{0}$. The term that contains the space variable yields upon repeated application of the binomial expansion:

$$
\left[\mathbf{s} \cdot \nabla_{\mathbf{s}_{0}}\right]^{k}=\left.\sum_{l=0}^{k} \sum_{m=0}^{l} C_{k}^{l} C_{l}^{m} s_{1}^{m} s_{2}^{l-m} s_{3}^{k-l}\left(\frac{\partial^{m}}{\partial s_{1}^{m}} \frac{\partial^{l-m}}{\partial s_{2}^{l-m}} \frac{\partial^{k-l}}{\partial s_{3}^{k-l}}\right)\right|_{\mathbf{s}_{=} \mathbf{s}_{0}}
$$

LEMMA 7.1. For $p+q+r \geq 1$

$$
\begin{gathered}
T_{3}^{1}\left[s_{1}^{p} s_{2}^{q} s_{3}^{r} \sum_{j=1}^{3} F_{j}^{\{n k l m\}}\left(\mathbf{s}_{0}\right) \frac{\partial H_{3}(\mathbf{s})}{\partial s_{j}}\right]=\frac{(-1)^{p+q+r}}{(p+q+r-1) !} \int_{-\infty}^{\sigma}(\sigma-u)^{p+q+r-l} \\
\times \sum_{j=1}^{3} F_{j}^{\{n k l m\}}\left(\mathbf{s}_{0}\right) \frac{\partial^{p+q+r}}{\partial \theta_{1}^{p} \partial \theta_{2}^{q} \partial \theta_{3}^{r}} \theta_{j} \frac{\partial \widehat{H}_{1, \boldsymbol{\theta}}(u)}{\partial u} d u
\end{gathered}
$$

is valid, where the indices $p, q$, and $r$ are positive integers or zero; $F_{j}^{\{n k l m\}}\left(\mathbf{s}_{0}\right)$ is a function that depends on the derivatives of $w_{j}(\mathbf{s})$ at the expansion point and on a set of integers $\{n k l m\}$ that determine exactly the order of the expansion and are such that they satisfy the inequalities $\infty>n \geq k \geq l \geq m \geq 0$.

Proof. In order to calculate the ST of a field $X_{3}(\mathbf{s})$ multiplied by an integer power of orie of the Cartesian coordinates, the following ST formulae must be used:

$$
T_{3}^{1}\left[\frac{\partial^{p+q+r} X_{3}(\mathbf{s})}{\partial s_{1}^{p} \partial s_{2}^{q} \partial s_{3}^{r}}\right]=\theta_{1}^{p} \theta_{2}^{q} \theta_{3}^{r} \frac{\partial^{p+q+r}}{\partial \sigma^{p+q+r}} \widehat{X}_{1, \boldsymbol{\theta}}(\sigma)
$$

and

$$
\begin{aligned}
\frac{\partial^{p+q+r} \widehat{X}_{1, \boldsymbol{\theta}}(\sigma)}{\partial \theta_{1}^{p} \partial \theta_{2}^{q} \partial \theta_{3}^{r}} & =(-1)^{p+q+r} \frac{\partial^{p+q+r}}{\partial \sigma^{p+q+r}} \int \delta(\sigma-\mathbf{s} \cdot \boldsymbol{\theta}) s_{1}^{p} s_{2}^{q} s_{3}^{r} X_{3}(\mathbf{s}) d \mathbf{s} \\
& =(-1)^{p+q+r} \frac{\partial^{p+q+r}}{\partial \sigma^{p+q+r}} T_{3}^{1}\left[s_{1}^{p} s_{2}^{q} s_{3}^{r} X_{3}(\mathbf{s})\right]
\end{aligned}
$$

The iterative integration of the last equation immediately implies that

$$
\begin{aligned}
T_{3}^{1}\left[s_{1}^{p} s_{2}^{q} s_{3}^{r} X_{3}(\mathbf{s})\right] & =(-1)^{p+q+r} \int_{-\infty}^{\sigma} d u_{p+q+r} \int_{-\infty}^{u_{p+q+r}} d u_{p+q+r-1} \cdots \int_{-\infty}^{u_{2}} d u_{1} \frac{\partial^{p+q+r} \widehat{X}_{1, \boldsymbol{\theta}}\left(u_{1}\right)}{\partial \theta_{1}^{p} \partial \theta_{2}^{q} \partial \theta_{3}^{r}} \\
& =\frac{(-1)^{p+q+r}}{(p+q+r-1) !} \int_{-\infty}^{\sigma} d u(\sigma-u)^{p+q+r-1} \frac{\partial^{p+q+r} \widehat{X}_{1, \boldsymbol{\theta}}(u)}{\partial \theta_{1}^{p} \partial \theta_{2}^{q} \partial \theta_{3}^{r}}
\end{aligned}
$$


By applying Eqs. (7.5) and (7.6), the following expression is derived for the head potential $H_{3}(\mathbf{s})$,

$$
\begin{array}{r}
(-1)^{p+q+r} \frac{\partial^{p+q+r}}{\partial \sigma^{p+q+r}} T_{3}^{1}\left[s_{1}^{p} s_{2}^{q} s_{3}^{r} \sum_{j=1}^{3} F_{j}^{\{n k l m\}}\left(\mathbf{s}_{0}\right) \frac{\partial H_{3}(\mathbf{s})}{\partial s_{j}}\right] \\
=\frac{\partial^{p+q+r}}{\partial \theta_{1}^{p} \partial \theta_{2}^{q} \partial \theta_{3}^{r}} \sum_{j=1}^{3} \theta_{j} F_{j}^{\{n k l m\}}\left(\mathbf{s}_{0}\right) \frac{\partial \widehat{H}_{1, \boldsymbol{\theta}}(\sigma)}{\partial \sigma} ;
\end{array}
$$

then, in light of Eq. (7.7), Eq. (7.8) leads to Eq. (7.4).

REMARK 7.1. Here it should be mentioned that the direction vector should be considered as an unconstrained vector when the partial derivatives of the direction vector $\theta$ are calculated, and, hence, its length should be allowed to vary. The form of the ST for an unconstrained direction vector in terms of the ST for a unit direction vector follows from the properties of the ST, $\widehat{H}_{1, \boldsymbol{\theta}_{u}}(\sigma)=\frac{1}{\left|\boldsymbol{\theta}_{u}\right|} \widehat{H}_{1, \boldsymbol{\theta}}\left(\frac{\sigma}{\left|\theta_{u}\right|}\right)$ and $\left|\boldsymbol{\theta}_{u}\right|=\sum_{j=1}^{3} \theta_{j}^{2}$.

With the help of the preceding analysis we are now ready to prove the following.

Proposition 7.1. Assuming the series expansion form of Eq. (7.1) for the log-conductivity gradient $\mathbf{w}(\mathbf{s})$, the application of the ST approach to the flow equation (6.1) leads to the one-dimensional series expansion

$$
\begin{aligned}
\frac{\partial^{2} \widehat{H}_{1, \theta}(\sigma)}{\partial \sigma^{2}} & +\sum_{n=0}^{\infty} \sum_{k=0}^{n} \sum_{l=0}^{k} \sum_{m=0}^{l} \frac{1}{n !} C_{n}^{k} C_{k}^{l} C_{l}^{m} \sum_{j=1}^{3} F_{j}^{\{n k l m\}}\left(\mathbf{s}_{0}\right) \\
& \times \frac{(-1)^{k}}{(k-1) !} \int_{-\infty}^{\sigma} d u(\sigma-u)^{k-l} \frac{\partial^{m}}{\partial \theta_{1}^{m}} \frac{\partial^{l-m}}{\partial \theta_{2}^{l-m}} \frac{\partial^{k-l}}{\partial \theta_{3}^{k-l}}\left[\frac{\theta_{j}}{\left|\theta_{u}\right|} \frac{\partial \widehat{H}_{1 . \boldsymbol{\theta}}\left(\frac{u}{\left|\theta_{u}\right|}\right)}{\partial u}\right]=0,
\end{aligned}
$$

where

$$
F_{j}^{\{n k l m\}}\left(\mathbf{s}_{0}\right)=\left.\left(\mathbf{s}_{0} \cdot \nabla\right)^{n-k}\left(\frac{\partial^{m}}{\partial s_{1}^{m}} \frac{\partial^{l-m}}{\partial s_{2}^{l-m}} \frac{\partial^{k-l}}{\partial s_{3}^{k-l}}\right) w_{j}(\mathbf{s})\right|_{s=s_{0}} .
$$

Proof. When the ST is applied to the flow Eq. (6.1), the first term readily transforms into

$$
T_{3}^{1}\left[\sum_{j=1}^{3} \frac{\partial^{2} H_{3}(\mathbf{s})}{\partial s_{j}^{2}}\right]=\sum_{j=1}^{3} \theta_{j}^{2} \frac{\partial^{2} \widehat{H}_{1, \boldsymbol{\theta}}(\sigma)}{\partial \sigma^{2}}=\frac{\partial^{2} \widehat{H}_{1, \boldsymbol{\theta}}(\sigma)}{\partial \sigma^{2}} .
$$

The second term consists of an infinite sum of terms which are proportional to the lefthand side of Eq. (7.4) of Lemma 7.1 above. Hence, using Eq. (7.4) together with Eq. (7.11) we obtain the desired Eq. (7.9).

If only the lowest term in the expansion is retained (0-th order approximation), the one-dimensional flow Eq. (7.9) is reduced to the following simple form:

$$
\frac{\partial^{2} \widehat{H}_{1, \boldsymbol{\theta}}^{(0)}(\sigma)}{\partial \sigma^{2}}+\sum_{j=1}^{3} w_{j}\left(\mathbf{s}_{0}\right) \theta_{j} \frac{\partial \widehat{H}_{1, \boldsymbol{\theta}}^{(0)}(\sigma)}{\partial \sigma}=0
$$


which admits the solution

$$
\frac{\partial \widehat{H}_{1 . \boldsymbol{\theta}}^{(0)}(\sigma)}{\partial \sigma}=h_{0}(\boldsymbol{\theta}) \exp \left[-\sigma \kappa_{\boldsymbol{\theta}}\left(\mathbf{s}_{0}\right)\right]
$$

where $\kappa_{\boldsymbol{\theta}}\left(\mathbf{s}_{0}\right)=\mathbf{w}\left(\mathbf{s}_{0}\right) \cdot \boldsymbol{\theta}$ and $h_{0}(\boldsymbol{\theta})=\frac{\partial \widehat{H}_{1, \boldsymbol{\theta}}^{(0)}(0)}{\partial \sigma}=-\widehat{J}_{1, \boldsymbol{\theta}}^{(0)}(0)$.

By applying the inverse ST to the above solution, an approximation of the threedimensional head potential in the neighborhood of the expansion point can be evaluated as

$$
H_{3}^{(0)}(\mathbf{s})=\frac{1}{2(2 \pi)^{2}} \int_{S_{3}} d \boldsymbol{\theta} h_{0}(\boldsymbol{\theta}) m^{(0)}(\sigma, \boldsymbol{\theta})
$$

where

$$
m^{(0)}(\sigma, \boldsymbol{\theta})=\kappa_{\boldsymbol{\theta}}\left(\mathbf{s}_{0}\right) \exp \left[-\kappa_{\boldsymbol{\theta}}\left(\mathbf{s}_{0}\right) \sigma\right] .
$$

In comparing the approximate Eq. (7.14) with the exact Eq. (6.23), it should be pointed out that Eq. (6.23) is a global solution with a range of validity that covers the flow domain, while the solution derived from the 0 -th order Taylor approximation is limited to a local description of the head potential.

However, the usefulness of the Taylor expansion method is not limited to the lowestorder approximation. Higher orders can be included by approximating the ST-1 of the head potential with a sequence of partial sums. For example, assuming that $\widehat{H}_{1 . \theta}(\sigma)$ is a continuous function defined on a closed domain, Weierstrass's theorem (e.g., [5]) states that it can be uniformly approximated by the following sequence:

$$
\widehat{H}_{1 . \boldsymbol{\theta}}(\sigma)=\lim _{M I \rightarrow \infty} \widehat{H}_{1 . \boldsymbol{\theta}}^{(M)}(\sigma)=\lim _{M \rightarrow \infty} \sum_{n, l . m}^{M} c_{n l m}^{(M)}(\boldsymbol{\theta}) \sigma^{n} .
$$

The unknown functions $c_{n l m}^{(M)}(\boldsymbol{\theta})$ in the above expansion can be expressed as a linear combination of spherical harmonics. The coefficients can be determined by canceling equal powers of $\sigma$ in the expansion of the flow equation.

8. Numerical approximations and stochastic moments solutions. The determination of the directional distribution $\widehat{J}_{1 . \boldsymbol{\theta}}(0)$ from the measurements will be discussed next, as well as certain numerical considerations regarding stochastic solutions of the $\operatorname{PDE}(6.1)$.

In hydrological applications an analytical expression for the log-conductivity random field is not usually available. Instead, discrete measurements or estimates of the logconductivity values are available at certain points. It is, therefore, assumed that a space grid $G_{\mathbf{z}}$, containing $N$ points at which the log-conductivity is known by measurements or estimation techniques, is embedded in the flow domain. The grid is said to cover the flow domain $D$, when all the points at the boundaries of the flow domain lie inside the boundaries of the grid. The number $N$ of grid points can be arbitrarily large, and it is in practice determined by computing considerations.

Following the introduction of the grid derivatives are replaced by finite differences and integrals by discrete summations over the grid. In order to simplify the analysis and 
without loss of generality, it is assumed that a covering grid composed of $N$ cubic cells of fixed size $b$ is embedded in the flow domain. Then, $\tilde{\kappa}_{\boldsymbol{\theta}}\left(u_{1}\right)$ can be approximated by replacing the integrals over planes with discrete summations as follows:

$$
\tilde{\kappa}_{\boldsymbol{\theta}}\left(u_{1}\right) \cong \frac{\sum_{\mathbf{z} \in G_{\mathbf{z}}}\left[K_{3}^{-1}(\mathbf{z}) \kappa_{\boldsymbol{\theta}}(\mathbf{z})\right] \delta_{1}\left(u_{1}-\mathbf{z} \cdot \boldsymbol{\theta}\right) \xi(\mathbf{z})}{\sum_{\mathbf{z} \in G_{\mathbf{z}}}\left[K_{3}^{-1}(\mathbf{z})\right] \delta_{1}\left(u_{1}-\mathbf{z} \cdot \boldsymbol{\theta}\right) \xi(\mathbf{z})}
$$

where $\xi(\mathbf{z})$ is a local integration weight that depends on the integration approximation scheme, and $\delta_{1}\left(u_{1}-\mathbf{z} \cdot \boldsymbol{\theta}\right)$ is the discrete summation resolution function that determines the grid points that are close enough to the plane $u_{1}-\mathbf{z} \cdot \boldsymbol{\theta}=0$ to be considered as "on the plane" points in the discrete approximation. Assuming that the increment of $u_{1}$ is given by the positive, real number $\Delta u_{1}$, the discrete summation resolution function is given by

$$
\delta_{1}\left(u_{1}-\mathbf{z} \cdot \boldsymbol{\theta}\right)= \begin{cases}1 & \text { if }\left|u_{1}-\mathbf{z} \cdot \boldsymbol{\theta}\right| \leq \frac{\Delta u_{1, i}}{2} \\ 0 & \text { otherwise. }\end{cases}
$$

The $H_{3}(\mathbf{s})$ can be approximated by the discrete average of the inverse ST $T_{1}^{3}$ head potential over $N_{\boldsymbol{\theta}}$ directions. In the case of a uniform distribution of azimuthal and polar angles the following is obtained:

$$
H_{3}(\mathbf{s}) \cong-\frac{1}{4 N_{\boldsymbol{\theta}}} \sum_{k=1}^{N_{\theta}} \widehat{J}_{1 . \boldsymbol{\theta}_{k}}(0) \sin \chi_{k} m\left(\mathbf{s} \cdot \boldsymbol{\theta}_{k} ; \boldsymbol{\theta}_{k}\right)
$$

at $\sigma_{k}=\mathbf{s} \cdot \boldsymbol{\theta}_{k}$, where $\chi_{k}$ is the angle between $\boldsymbol{\theta}_{k}$ and the $s_{3}$-axis. The inversion formula should be modified for a different distribution of the direction vectors. For example, see [11].

Equation (8.3) determines the head potential for every $\mathrm{RF}$ realization in terms of the one-dimensional negative head slope at the origin for each of the sampled directions $\boldsymbol{\theta}_{k}$. However, the value of the derivative of the ST for each direction $\boldsymbol{\theta}_{k}$ requires the values of the gradient of $H_{3}(\mathbf{s})$ on the plane that passes through the origin and is perpendicular to $\boldsymbol{\theta}_{k}$. This boundary condition on $H_{3}(\mathbf{s})$ is unrealistic in practical problems. Essentially, it is required to determine the directional distribution so that the solution will be consistent with the three-dimensional flow equation.

With the exception of certain specific cases, there is not at the moment a general method that allows a direct calculation of the directional distribution. It is possible, of course, to test different hypotheses by applying the inverse ST to obtain the three-dimensional head solution and by testing whether the solution satisfies the threedimensional flow equation. Fortunately, as regards discrete approximations in practice there is a method that allows a straightforward calculation of the $\widehat{J}_{1, \boldsymbol{\theta}_{k}}(0)$, as follows: (i) It is assumed that measurements of $H_{3}(\mathbf{s})$ are available at $N_{\boldsymbol{\theta}}$ points within the flow domain. (ii) When these values are substituted in the corresponding equation for the mean of $H_{3}(\mathbf{s})$ they yield a linear system of $N_{\boldsymbol{\theta}}$ equations with $N_{\boldsymbol{\theta}}$ unknowns (i.e., the boundary values $\left.\widehat{J}_{1, \theta_{k}}(0)\right)$. (iii) Solution of the system leads to the determination of $\widehat{J}_{1 . \theta_{k}}(0)$. It is also possible to have boundary conditions that involve both values of the hydraulic head and its gradient. For example, in hydrological applications mixed Dirichlet and Neuman boundary conditions are routinely used. In such a case the system (8.3) 
should be augmented by a respective system of equations for the hydraulic gradient. Preliminary numerical calculations indicate that the system (8.3) is unstable when the number of equations is equal to the number of direction vectors. This singularity can be removed by means of the singular value decomposition method by using an overdetermined system, i.e., specifying the boundary condition at a number of points higher than the number of direction vectors.

Just as for the RF realizations, the inverse ST can also be used to derive the corresponding flow moment solutions, namely the spatial mean $\bar{H}_{3}(\mathbf{s})$ and the spatial covariance $c_{H}\left(\mathbf{s}, \mathbf{s}^{\prime}\right)=\overline{H_{3}(\mathbf{s}) H_{3}\left(\mathbf{s}^{\prime}\right)}$ of the head potential (the bar denotes stochastic averaging; see, e.g., [9]). More specifically, assuming that the boundary conditions have been determined, the second-order statistics of the head potential can be calculated by

$$
\bar{H}_{3}(\mathbf{s}) \cong-\frac{1}{4 N_{\boldsymbol{\theta}}} \sum_{k=1}^{N_{\theta}} \widehat{J}_{1, \boldsymbol{\theta}_{k}}(0) \sin \chi_{k} \overline{m\left(\mathbf{s} \cdot \boldsymbol{\theta}_{k} ; \boldsymbol{\theta}_{k}\right)}
$$

and

$$
c_{H}\left(\mathbf{s}, \mathbf{s}^{\prime}\right) \cong \frac{1}{16 N_{\boldsymbol{\theta}}^{2}} \sum_{k=1}^{N_{\theta}} \sum_{j=1}^{N_{\theta}} \widehat{J}_{1 . \boldsymbol{\theta}_{j}}(0) \sin \chi_{k} \sin \chi_{j} c_{m}\left(\mathbf{s}, \mathbf{s}^{\prime} ; \boldsymbol{\theta}_{k}, \boldsymbol{\theta}_{j}\right),
$$

where $\overline{m\left(\mathbf{s} \cdot \boldsymbol{\theta}_{k} ; \boldsymbol{\theta}_{k}\right)}$ and $c_{m}\left(\mathbf{s}, \mathbf{s}^{\prime} ; \boldsymbol{\theta}_{k}, \boldsymbol{\theta}_{j}\right)$ are, respectively, the mean and the covariance functions of the directional head generator $(6.22)$ calculated by

$$
\overline{m\left(\mathbf{s} \cdot \boldsymbol{\theta}_{k} ; \boldsymbol{\theta}_{k}\right)} \cong \frac{1}{N_{M}} \sum_{r=1}^{N_{M}} m_{r}\left(\mathbf{s} \cdot \boldsymbol{\theta}_{k} ; \boldsymbol{\theta}_{k}\right)
$$

and

$$
c_{m}\left(\mathbf{s}, \mathbf{s}^{\prime} ; \boldsymbol{\theta}_{k}, \boldsymbol{\theta}_{j}\right) \cong \frac{1}{N_{M}} \sum_{r=1}^{N_{M}} m_{r}\left(\mathbf{s} \cdot \boldsymbol{\theta}_{k} ; \boldsymbol{\theta}_{k}\right) m_{r}\left(\mathbf{s}^{\prime} \cdot \boldsymbol{\theta}_{i} ; \boldsymbol{\theta}_{i}\right),
$$

where $N_{M}$ is the number of RF realizations used. Computational issues will be further investigated in a forthcoming publication.

\section{REFERENCES}

[1] I. Bialynicki-Birula, M. Cieplak, and J. Kaminski, Theory of Quanta, Oxford University Press, New York, N. Y., 1992

[2] G. Christakos, The space transformations and their applications in systems modelling and simulation, Proc. 12th Intern. Confer. on Modelling and Simulation (AMSE) 1 (3), Athens, Greece, 1984, pp. $49 \cdot 68$

[3] G. Christakos, Random Field Models in Earth Sciences, Academic Press, San Diego, CA, 1992

[4] G. Christakos and D. T. Hristopulos, Stochastic space transformation techniques in subsurface hydrology-Part 2: Generalized spectral decompositions and Plancherel representations, Stochastic Hydrology and Hydraulics 8, no. 2, 117-138 (1994)

[5] R. Courant and D. Hilbert, Methods of Mathematical Physics, Vol. I, J. Wiley, New York, N. Y., 1953

[6] I. M. Gel'fand and G. E. Shilov, Generalized Functions, Vol. 1, Academic Press, New York, N. Y., 1964

[7] S. Helgason, The Radon Transform, Birkhauser, Boston, Basel, Stuttgart, 1980

[8] F. John, Plane Waves and Spherical Means, Springer-Verlag. New York, N. Y., 1955

[9] M. Loeve, Probability Theory, Van Nostrand, Princeton, 1953

[10] A. Scheidegger, Physics of Flow Through Porous Media, University of Toronto Press, Toronto, Canada, 1960

[11] A. K. Jain, Fundamentals of Digital Image Processing, Prentice Hall. Englewood Cliffs, NJ, 1989 